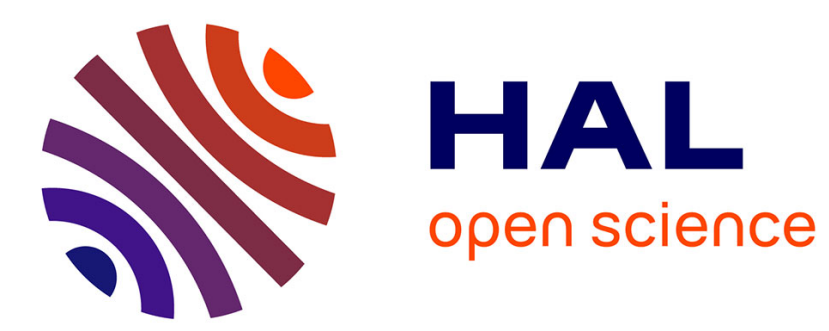

\title{
La science et la doctrine juridiques à l'épreuve de la polysémie des concepts
}

Boris Barraud

\section{To cite this version:}

Boris Barraud. La science et la doctrine juridiques à l'épreuve de la polysémie des concepts. Revue Interdisciplinaire d'Etudes Juridiques, 2016, 76, p. 5 s. hal-01367484

\section{HAL Id: hal-01367484 https://hal-amu.archives-ouvertes.fr/hal-01367484}

Submitted on 16 Sep 2016

HAL is a multi-disciplinary open access archive for the deposit and dissemination of scientific research documents, whether they are published or not. The documents may come from teaching and research institutions in France or abroad, or from public or private research centers.
L'archive ouverte pluridisciplinaire HAL, est destinée au dépôt et à la diffusion de documents scientifiques de niveau recherche, publiés ou non, émanant des établissements d'enseignement et de recherche français ou étrangers, des laboratoires publics ou privés. 
Aix $*$ Marseille université

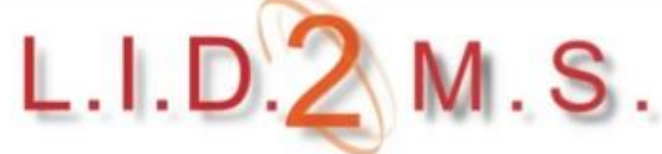

Laboratoire Interdisciplinaire de Droit des Medias \& des Wutations Sociales

Boris Barraud, "La science et la doctrine juridiques à l'épreuve de la polysémie des concepts", Revue Interdisciplinaire d'Études Juridiques 2016, n 76, p. 5 s.

manuscrit de l'auteur

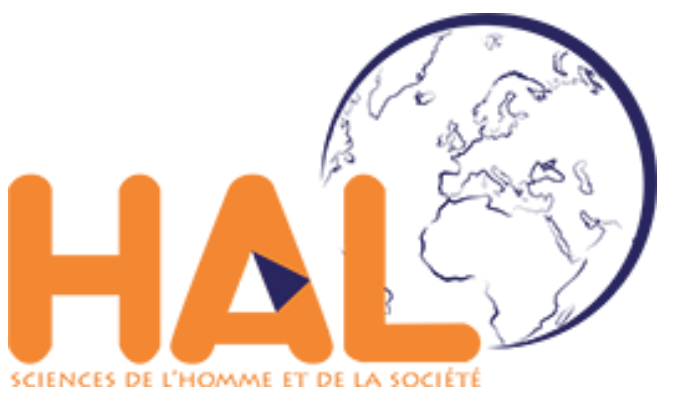




\title{
I. Les concepts monosémiques et la scientificité du discours doctrinal
}
A. L'utilisation des concepts, marque de la doctrine juridique
B. La précision des concepts, exigence de la science juridique

\section{Les concepts polysémiques et l'ascientificité du discours doctrinal contemporain}
A. L'usage de concepts polysémiques, propre de la doctrine juridique contemporaine
B. La lutte contre les concepts polysémiques, enjeu de la science juridique contemporaine

\begin{abstract}
"Mal nommer les choses, écrivait Albert Camus, c'est ajouter aux malheurs du monde ». En cette étude, est soutenu que "mal nommer les choses juridiques, c'est ajouter aux malheurs du monde juridique ». L'ambition est de souligner combien le développement de l'usage de concepts polysémiques peut être préjudiciable à la qualité scientifique des analyses doctrinales et, en conséquent, combien l'attachement aux termes et expressions monosémiques est une donnée cardinale pour l'idée de "science » juridique. Si l'incertitude quant aux signifiés désignés par les signifiants est parfois un instrument utile du point de vue des législateurs, les commentateurs et penseurs du droit, eux, doivent s'efforcer de recourir à un arsenal conceptuel rigoureux et précis, n'obligeant le lecteur ou auditeur à aucune interprétation. L'enjeu devient capital à l'aube de l'avènement d'un droit et d'une culture juridiques dits "postmodernes » qui invitent à refondre en partie les outils "modernes » d'étude et de pensée du droit.
\end{abstract}


Le droit, sans le verbe qui l'exprime, est nu. Les législateurs ou jurislateurs, chargés de produire les règles et institutions positives, autant que la doctrine, dont la tâche est de décrire et d'expliquer l'œuvre des législateurs, seraient démunis s'ils n'avaient plus à disposition leur principal outil de travail : le langage juridique. Oscar Wilde écrivait : «Nos pensées naissent tout habillées de mots » ${ }^{1}$. Mais, bien au contraire, lorsqu'elles naissent, les pensées sont nues et, ensuite, qui veut les communiquer - ou, mieux, les transmettre - doit les habiller de mots. Cela vaut, évidemment, à l'égard des pensées juridiques. Si le droit peut être l'objet d'une science, cette dernière ne saurait revêtir les mêmes atours que la chimie ou la physique ; elle est une science verbale qui étudie des ensembles verbaux au moyen d'autres ensembles verbaux. Aussi la scientificité des travaux des juristes est-elle, dans une large mesure, dépendante de la précision et de la non-équivocité de ces derniers. Comme «e $=\mathrm{mc}^{2} »$ exprime de façon claire et précise une vérité scientifique, les propositions de la doctrine juridique quant à ce qu'est le droit, comment est le droit, pourquoi est le droit et, éventuellement, que doit être le droit doivent être claires et précises, dépourvues d'ambiguïtés. Il incombe aux juristes de recourir à un langage au caractère monosémique et non polysémique, c'est-à-dire que les signifiants employés doivent s'attacher à un signifié unique et non à des signifiés potentiellement multiples. Les descriptions et explications faites de mots qui ont la «propriété de renvoyer à plusieurs signifiés présentant des traits sémantiques communs $»^{2}$ portent une moindre scientificité que celles qui se composent d' "unités significatives, lexicales ou grammaticales, qui n'ont qu'un seul sens $»^{3}$.

S'enquérir de la problématique de la polysémie dans le discours de la doctrine juridique, c'est mettre l'accent à la fois sur la «manière de dire » et sur la «matière du dire ». Montaigne séparait strictement l'une et l'autre et concentrait toute son attention sur la «manière de dire ${ }^{4}$; mais la manière est peu de chose sans la matière, la forme paraît vide sans le fond qui la remplit ; toute forme n'est utile que lorsqu'elle est au service d'un fond. Un beau mot l'est moins parce qu'il « sonne » ou « résonne » ${ }^{5}$, que parce qu'il signifie, et qu'il signifie univoquement. Tel est, en résumé, le propos de la présente étude, étant ajouté que de la signification univoque découle la scientificité - et étant précisé que «la science du droit » ici en cause

${ }^{1}$ Cité par A. Dulot, Ce que penser veut dire, L’Harmattan, coll. Pour comprendre, 2013, p. 86.

${ }^{2} \mathrm{~V}^{\circ}$ « Polysémie », in Trésor de la langue française.

${ }^{3} \mathrm{~V}^{\circ} \ll$ Monosémie », in Trésor de la langue française.

${ }^{4}$ M. DE Montaigne, Les essais, 1588, L. III, chap. 8 ( « Le monde n’est qu’une école de recherche. Dans ces exercices, ce n'est pas à qui atteindra le but mais à qui ferra les plus belles courses. Autant peut faire le saut celui qui dit vrai que celui qui dit faux. Car ce qui est en cause pour nous, c'est la manière de dire, non la matière. [...] Ce qui compte n'est pas le résultat mais la démarche qui y conduit »).

${ }^{5}$ F. HÉRITIER, Le goût des mots, Odile Jacob, 2013. Néanmoins, l'auteur souligne que certains mots voient leurs sens particulièrement bien exprimés par leurs sonorités. Et de citer, par exemple, «éternuer»et « explosif». Mais, à l'inverse, la sonorité de " procrastiner » laisse entendre que ce verbe signifierait « dire des injures ou invectiver », ce qui ne correspond guère à son sens communément admis (ibid.). 
n'est bien sûr qu'une conception de la science du droit (celle défendue par l'auteur de ces lignes et qui se résume dans les principes d'objectivité et d'empirisme) et en aucun instant une réalité donnée ou même une vérité absolue et indiscutable -

Le juriste, qu'il se borne à décrire — et, nécessairement, à interpréter — le droit positif ou qu'il cherche à le comprendre, à l'expliquer ou à l'influencer, recourt à des méthodes particulières composées de logiques, de modes de raisonnement, de classifications, de techniques d'expression, mais aussi de concepts, c'est-à-dire de mots-clés ou mots-cadenas permettant, mieux que de longues tirades, d'exprimer en un instant une ou même plusieurs idée(s)-force(s). La méthodologie juridique repose ainsi sur une terminologie et sur un arsenal conceptuel qui doivent être « riches » mais aussi «précis ${ }^{1}$. S'il est vrai - mais cela est loin d'être assuré — que la méthode des juristes est la «méthode scientifique unique qui s'impose pour toutes les sciences : [...] celle des sciences naturelles $»^{2}$, alors il incombe bien aux commentateurs et penseurs du droit de s'appuyer sur une structure langagière et sémantique aussi développée et complète que stricte et rigoureuse.

Par suite, les moyens et les méthodes autant que les perspectives et les objectifs des juristes varient inéluctablement selon qu'il s'agit d'élaborer la loi, de rendre un jugement, de négocier un contrat ou d'exposer, expliquer et enseigner le droit. En ces lignes, est principalement envisagé le juriste qui observe le droit de l'extérieur, sans prendre part à sa construction ou à son évolution, si ce n'est indirectement en tant que source matérielle, c'est-à-dire le professeur, l'homme de doctrine. Ce dernier, plus encore que les autres, recourt aux concepts afin d'exprimer de la manière la plus limpide et rapide sa perception et sa conception des phénomènes juridiques. Il est plus périlleux pour un législateur de recourir à ces raccourcis sémantiques que sont les concepts, quoique, pour autant, il ne s'en prive souvent pas. Lesdits phénomènes juridiques ne se limitent pas à des accumulations plus ou moins ordonnées de règles positives. Ils contiennent au moins autant de mouvements de savoirs sur et autour des règles positives. Ce sont ces contenus épistémiques méta-positifs qui sont ici en cause, car ils sont davantage concernés par la question de la scientificité que les savoirs des professionnels-praticiens du droit, lesquels consistent en des savoir-faire plus qu'en des savoir-savoir, en des savoirs techniques plus qu'en des savoirs scientifiques — même si les uns ne sont pas exclusifs des autres -

On observe fréquemment que chacun des auteurs qui composent la doctrine juridique possède à la fois un style littéraire et un style scientifique. Sans doute le premier doit-il passer au second plan, si bien que celui qui possède un grand style scientifique mais un petit style littéraire serait meilleur commentateur du droit que celui qui possède un grand style littéraire mais un petit style scientifique - cela évidemment aux yeux de l'auteur de ces lignes qui défend la scientificité du droit

${ }^{1}$ J.-L. Bergel, «Méthodologie juridique », in D. Alland, S. Rials, dir., Dictionnaire de la culture juridique, Lamy-Puf, coll. Quadrige-dicos poche, 2003, p. 1021.

${ }^{2}$ M. TROPER, «Science du droit», in Dictionnaire de la culture juridique, op. cit., p. 1394 (non souligné dans le texte original). 
compris comme discipline académique et non de manière absolue, des courants tels que «droit et littérature » s'attachant à de tout autres conceptions de ce droit C'est le style scientifique qui l'emporte dès lors que les facultés de droit s'envisagent comme des écoles chargées de former des scientifique et non d'engendrer des hommes de lettres ou des artistes; même si, évidemment, qui possède un grand style scientifique et un grand style littéraire, à l'instar d'un Carbonnier, est sans doute un très grand commentateur du droit. Toutefois, à la différence d'autres domaines scientifiques au sein desquels un langage spécifique, quasi-indépendant de la langue commune, s'impose au chercheur qui ne dispose d'aucune marge de manœuvre lexicale, le juriste recourt, la plupart du temps, à cette langue commune, si bien qu'il lui est plus facile de se faire comprendre du profane que cela n'est le cas concernant le mathématicien ou l'astrophysicien. Là où les chiffres, les calculs, les symboles et les schémas sont les instruments de ces derniers, les mots, plus ou moins courants, sont les outils du commentateur et penseur du droit. Partant, il peut aisément, s'il le souhaite, orner son propos de divers artifices qui, s'ils touchent peu au fond de l'explication, sont en mesure de modifier radicalement sa forme. En ce sens, il n'est peut-être pas exagéré de dire que «le cours magistral du professeur de droit tient de la prédication et du théâtre ${ }^{1}$. Nombreux sont les auteurs-juristes qui, à raison, songent, lorsqu'ils écrivent, à «l'ennui du lecteur qui est facilement découragé par les abstractions ou la sécheresse du discours $»^{2}$. On ne se félicite jamais qu'une analyse soit rédigée de manière complexe et absconse ; en revanche, on félicite régulièrement qui adopte un style simple et fluide, ne contraignant pas le lecteur à lire puis relire les mêmes phrases.

Les qualités formelles du discours sont assurément importantes du point de vue de qui aspire à être écouté et entendu ; mais elles ne peuvent prospérer sans quelques qualités substantielles concomitantes. Plus encore, certains écrits restent dans la mémoire du droit parce qu'ils ont exprimé une ou plusieurs idée(s) fondatrice(s), mais aucun n'accède à la notoriété uniquement en vertu de ses seuls mérites formels. La valeur matérielle paraît donc être d'un poids supérieur par rapport à la valeur formelle dans la pesée de la valeur absolue des travaux jus-scientifiques, ce qui n'implique bien sûr pas que la forme soit de nulle importance. Entre matière et manière, s'il fallait choisir - mais il ne le faut certainement pas puisqu'une étude de qualité est un bon fond derrière une belle forme - la matière devrait l'emporter. Et si la thématique de la polysémie des concepts juridiques se situe à cheval entre problématique formelle et problématique matérielle, elle s'incline néanmoins en direction de cette dernière. Un concept peut être pertinent formellement, parce qu'il permet, comme un intitulé, de dire beaucoup en peu de mots, mais son utilisation se justifie principalement, en théorie, sous l'angle substantiel, en ce qu'il sert à

${ }^{1}$ J.-M. CARBASSE, «Professeurs à la faculté de droit », in Dictionnaire de la culture juridique, op. cit., p. 1245.

${ }^{2}$ G. THUILLIER, «Obsolescence des travaux juridiques », in Dictionnaire de la culture juridique, op. cit., p. 1102. 
exprimer un principe ou une notion cardinale. Or, s'il n'exprime pas un principe ou une notion capitale mais potentiellement plusieurs principes ou notions différentes, obligeant le lecteur à faire œuvre interprétative, il perd une large part de sa qualité significative et, dès lors, de sa qualité scientifique. Paradoxalement, il ne dit alors non pas beaucoup mais peu.

Il ne paraît donc pas juste de retenir que, en droit, « les mots importent peu » ${ }^{1}$. Au contraire, le droit n'est que mots ; sans mots, il n'y aurait pas plus de droit et de science du droit que de poésie et de littérature. Les mots sont comme la partie émergée d'un iceberg; ils sont la manifestation et le moyen d'action du droit. Le système juridique n'existe qu'à travers les locutions qui lui donnent corps ; simple squelette, il serait tout aussi inconsistant qu'inopérant et inefficace. Le droit peut se concevoir telle une science ; il est peut-être aussi, dans une certaine mesure, un art plus ou moins assumé ; et on relève que ce n'est que depuis le $\mathrm{XIX}^{\mathrm{e}}$ siècle que l'idée romaine, affirmée notamment par Ulpien, d' «art du droit » a été subrogée par celle de «science du droit $»^{2}$. L'art du droit n'est d'ailleurs pas forcément incompatible avec la science du droit et avec l'exigence de monosémie des concepts puisque la précision peut être le fait d'un art. Mais les juristes ne sauraient être assimilés à des poètes, soit à des écrivains qui souvent privilégient la forme par rapport au fond ou qui, du moins, souvent cherchent à séduire par la forme plutôt que par le fond, la forme valant alors par elle-même autant qu'en tant que servante du fond. On note ainsi que le mariage imaginé de la poésie et du droit est "étonnant» et qu' "il aurait certainement suscité les doutes de Corneille [...] pour qui les hommes de loi ne sont que de tristes laquais au service du pouvoir» et le «scepticisme de nombreux juristes d'aujourd'hui pour qui le terme de poète désigne un esprit rêveur, étranger à la rigueur et à la précision du droit $»^{3}$. Le monde juridique est certainement une esthétique et une harmonie, donc une poésie, mais cela est secondaire par rapport aux enjeux pratiques et concrets qui l'imprègnent. Les écrits juridiques doivent être «élégants », mais l'élégance n'est qu'une qualité parmi d'autres, et ils doivent en premier lieu revêtir les qualités d' «expressivité », d'《unité » et de «systématicité » ${ }^{4}$.

Le droit est avant toute autre chose une pratique ; et la praticabilité du droit dépend spécialement de la praticabilité de son langage. Les mots du droit doivent être des mots clairs et précis, ne laissant aucune place à l'incertitude ou, en tout cas, le moins de place possible à l'incertitude puisque l'ouverture du langage interdit

${ }^{1}$ J.-P. ChaZAL, «Philosophie du droit et théorie du droit, ou l'illusion scientifique », Arch. phil. droit 2001, p. 328.

${ }^{2}$ A. LECA, La genèse du droit - Essai d'introduction historique au droit, $3^{\mathrm{e}}$ éd., Librairie de l'Université d'Aix-en-Provence, 2002, p. 11.

${ }^{3}$ A. LECA, La lyre de Thémis ou la poésie du droit, Presses universitaires d'Aix-Marseille, 2011, p. 18. Cependant, l'auteur observe à juste titre que, comme le notait déjà Pascal, « on ne sait pas en quoi consiste l'agrément qui est l'objet de la poésie » (ibid., p. 24).

${ }^{4}$ M. XIFARAS, «La Veritas Iuris selon Raymond Saleilles - Remarques sur un projet de restauration du juridisme » Droits 2008, n 47, p. 77 s. 
d'imaginer que les sens de certains termes et expressions pourraient se laisser saisir sans interprétation aucune. S'il va de soi que la réalité de l'expression juridique n'est pas tout à fait celle-là, a fortiori dès lors que le législateur recourt parfois volontairement à des notions floues afin de se délester au profit des interprètes officiels que sont les juges, il lui incombe de tendre vers cet idéal, notamment quant à son pan doctrinal. Nicolas Boileau enseignait que « ce qui se conçoit bien s'énonce clairement, et les mots pour le dire viennent aisément $»^{1}$. Or il semble que les bouleversements ou, moins fortement, les mutations qui imprègnent les phénomènes juridiques à l'heure du $\mathrm{XXI}^{\mathrm{e}} \mathrm{s}$. ont pour effet de rendre ceux-ci moins aisément intelligibles. Dès lors, «ce qui se conçoit mal s'énonce obscurément, et les mots pour le dire viennent difficilement ». Cela interroge la scientificité de la « science » juridique, laquelle — telle que la conçoit l'auteur de ces lignes du moins — ne peut faire l'économie de l'usage d'un ensemble relativement complet de concepts clairs et précis, de concepts univoques et monovalents. La connaissance est la mise en relation d'un sujet et d'un objet par le truchement d'une structure opératoire ${ }^{2}$. L'irruption de la polysémie casse cette relation et, partant, ébranle la gnoséologie juridique et met en péril la science du droit.

La présente étude, consacrée au danger de la polysémie pour la scientificité des travaux doctrinaux, relève sans doute de l'épistémologie juridique en ce qu'elle entend participer de la «connaissance de la connaissance ${ }^{3}$ et en ce qu'elle « n'a pas pour objet le droit mais la connaissance du droit » et s'efforce « d'apprécier les moyens que les juristes emploient pour connaître le droit $»^{4}$. Elle s'attache également à la sémiotique juridique, soit à l'étude des systèmes de signification juridiques - les rapports entre des signes et des sens — et de mise en lien de la pensée juridique et de la réalité juridique ${ }^{5}$, ainsi qu'à la linguistique juridique qui est, plus simplement, l'étude de la langue juridique ${ }^{6}$. Enfin, elle s'inscrit aussi dans le cadre de la méthodologie juridique, laquelle «s'attache aux instruments et mécanismes de conception, de compréhension et d'application du droit » et particulièrement aux «concepts [...] de l'appareil juridique ${ }^{7}$. Son objet est de rechercher, si ce n'est de démontrer, combien les concepts, outils essentiels à la doctrine juridique, doivent être monosémiques (I) et combien le développement contemporain de l'usage de concepts polysémiques est une menace pour la légitimité scientifique des commentaires, analyses et explications produits par ladite doctrine (II). L'enjeu n'est pas anodin puisque « la compréhension est la manière

\footnotetext{
${ }^{1}$ N. BoILEAU, L'art poétique, 1674, Chant $\mathrm{I}^{\mathrm{er}}$.

${ }^{2}$ J. Piaget, Logique et connaissance scientifique, $2^{\mathrm{e}}$ éd., Gallimard, coll. Encyclopédie de la Pléiade, 1986.

${ }^{3}$ Réf. à E. Morin, La Méthode - t. III : Connaissance de la connaissance, Le Seuil, 1992.

${ }^{4}$ Ch. Atias, Épistémologie juridique, Puf, 1985, p. 18.

${ }^{5}$ M. Olivier, Peirce : la pensée et le réel, Hermann, 2013.

${ }^{6} C f$. G. CORNU, Linguistique juridique, 3 éd., Montchrestien, coll. Domat droit privé, 2005.

${ }^{7}$ J.-L. Bergel, « Méthodologie juridique », in Dictionnaire de la culture juridique, op. cit., p. 1023.
} 
pour l'homme de se réconcilier avec la réalité $»^{1}$. Entre le droit pensé et le droit réel, seul le second possède un véritable intérêt pratique et, partant, est le «véritable droit », celui qui intéresse la grande majorité des juristes. Comme l'écrivait Duguit, « une théorie juridique n'a de valeur que si elle met en œuvre des faits réels $»^{2}$. Or le droit pensé, s'il est abscons, amphigourique et sujet à interprétation, ne peut être mieux qu'une compréhension très imparfaite du droit réel.

\section{Les concepts monosémiques et la scientificité du discours doctrinal}

L'œuvre d'Hugo Grotius est une illustration pertinente de la rigueur qui doit accompagner les développements scientifiques de la doctrine. L'auteur du Droit de la guerre et de la paix plaçait ses travaux sous le signe d'un rationalisme systématique exigeant clarté des principes et rigueur de la démarche. Il écrivait notamment : «Pour ce qui est du style, je n'ai pas voulu, en ajoutant une abondance de paroles à la multitude des choses à traiter, causer du dégoût au lecteur, dont j'avais en vue l'intérêt. Aussi ai-je employé autant que je l'ai pu le style concis qui convient à l'enseignement ». La simplicité et la clarté du propos sont assurément à rechercher, ce qui justifie que les juristes convoquent abondamment les concepts ; mais encore faut-il que ces concepts ne soient pas polysémiques. C'est pourquoi Grotius s'efforçait de toujours proposer des développements définitionnels substantiels afin de fixer sans ambiguïté le sens des principales notions utilisées.

Durkheim remarquait que, «tandis que le physicien qui étudie la nature a le sentiment très vif des résistances qu'elle lui oppose [...], il semble que le sociologue se meuve au milieu de choses immédiatement transparentes pour l'esprit, tant est grande l'aisance avec laquelle on le voit résoudre les questions les plus obscures ${ }^{3}$. Le juriste, à ce point de vue, ressemble de plus en plus au physicien et, paradoxalement, de moins en moins au sociologue. Aussi paraissait-il opportun d'ainsi insister, au-delà de l'intérêt indiscutable de disposer de concepts (A), sur l'intérêt généralement lui-aussi indiscutable de disposer de concepts aux signifiés clairs et précis, donc aisément intelligibles (B). Les intellectuels, et les penseurs du droit tout spécialement, sont, pour reprendre des mots de Raymond Aron, «ivres de concepts ${ }^{4}$. Par suite, il est décisif qu'ils s'abreuvent de concepts utiles et pertinents, soit de concepts monosémiques qui, seuls, paraissent être des concepts

${ }^{1}$ H. ARENDT, La crise de la culture (1968), trad. P. Lévy, Gallimard, coll. Folio essais, 2011, p. 17.

${ }^{2}$ L. Duguit, Traité de droit constitutionnel - Tome deuxième : La théorie générale de l'État - Première partie : Éléments, fonctions et organes de l'État, $3^{\mathrm{e}}$ éd., De Boccard, 1928, p. 87.

${ }^{3}$ É. Durkheim, Les règles de la méthode sociologique, $2^{\mathrm{e}}$ éd. (1937), Puf, coll. Bibliothèque de philosophie contemporaine, 1973, p. XIV-XV.

${ }^{4}$ Cité par cité par S. LAUNAY, «La théorie réaliste est-elle morte pour cause de "mondialisation" ? ", in G. Demuijnck, P. Vercauteren, dir, L'État face à la globalisation économique - Quelles formes de gouvernance?, Sandre, 2009, p. 75. 
scientifiques, c'est-à-dire des concepts dont le sens est objectivement et empiriquement saisissable. Jhering, à la fin du XIX ${ }^{\mathrm{e}}$ s., regrettait que « la science du droit tourne en mathématique $»^{1}$. En ces pages, est soutenu que plus la science du droit se rapprochera de la rigueur des mathématiques, plus elle méritera ce nom de «science du droit » et moins elle pourra être contestée - encore une fois, cela tout en n'ignorant pas qu'est ici en cause une acception de la science du droit et non « la » science du droit universelle et essentialisée -

\section{A. L'utilisation des concepts, marque de la doctrine juridique}

La pensée peut être limpide, « les mots pour le dire » ${ }^{2}$ sont souvent hésitants et incertains, risquant de la travestir ou même de la trahir. Ainsi que le relevait Wittgenstein, les idées ne posent guère de difficulté tant qu'elles demeurent dans l'esprit, mais il en va autrement dès qu'il faut leur donner forme écrite ${ }^{3}$. Les mots sont une technique d'expression de la pensée et non la pensée elle-même. Un concept se conçoit déjà psychiquement, puis il doit être «traduit " ${ }^{4}$, sans quoi, quelles que soient ses qualités matérielles, il resterait confidentiel, comme un beau tableau que le peindre conserverait dans son grenier. Et Gilles Deleuze et Félix Guattari d'expliquer que, malheureusement, la pensée et son contenu représentationnel, le plus souvent, ne s'accordent pas exactement, sont diffractés ${ }^{5}$. Si tel n'était pas le cas, il suffirait de penser pour connaître. Mais « le réel est d'une étoffe qu'aucun mot ne peut saisir ${ }^{6}$; et «toute proposition, enseignait Duguit, est une abstraction puisqu'elle est l'expression non pas de la réalité, mais de l'idée que nous nous formons de la réalité ${ }^{7}$. Le vocabulaire est donc fait d'étiquettes qui ne laissent entrevoir qu'imparfaitement les choses qu'elles enferment; et le vocabulaire scientifique n'échappe qu'un peu à la règle. Reste alors à chercher les mots susceptibles de décrire le moins imparfaitement le réel, peu important que ce réel soit un réel physique et tangible ou un réel psychologique et cognitif. Aussi les

${ }^{1}$ R. VON JHERING, Le combat pour le droit, 1875.

${ }^{2}$ Réf. à M. CARDINAL, Les mots pour le dire, Le livre de poche, 1977.

${ }^{3}$ L. Wittgenstein, Tractatus logico-philosophicus, trad. P. Klossowski, Gallimard, coll. Tel, 2001.

${ }^{4}$ F. GÉNY, Science et technique en droit privé positif, t. IV, Librairie du Recueil Sirey, 1924, p. 123. La «traduction» ici en cause n'a rien à voir avec la «traduction juridique » comprise comme traduction d'un concept juridique ou d'une autre donnée juridique d'une langue vers une autre (C. BOCQUET, La traduction juridique - Fondement et méthode, De Boeck, coll. Traducto, 2008). Il y aurait beaucoup à dire sur le lien entre traduction et polysémie dès lors que, par exemple, le terme latin «lex» a été traduit en français par «loi » et en anglais par «law», «law» se traduisant en français non par «loi» mais par « droit».

${ }^{5}$ G. Deleuze, F. Guattari, Mille plateaux - Capitalisme et schizophrénie 2, Minuit, coll. Critique, 1980.

${ }^{6}$ H. Bergson, Essai sur les données immédiates de la conscience, 10 éd., Puf, coll. Quadrige, 2013, p. 7.

${ }^{7}$ L. Dugurt, Traité de droit constitutionnel - Tome deuxième : La théorie générale de l'État-Première partie : Éléments, fonctions et organes de l'État, $3^{\mathrm{e}}$ éd., De Boccard, 1928, p. 87. 
concepts - peut-être aux côtés des classifications - sont-ils les instruments les plus indispensables à la connaissance et à l'expression scientifique, quoique cela soit plus ou moins évident selon la science en cause. Les sciences humaines et sociales ont, plus que les sciences de la nature, besoin de se développer au travers de concepts ; et la science juridique, plus que les autres sciences humaines et sociales, recourt aux concepts afin de comprendre et expliquer le droit positif, sa logique, sa structure et son évolution. Son objet, en effet, n'est pas connaissable immédiatement; seule une connaissance médiatisée est envisageable et c'est pourquoi posséder un attirail conceptuel perfectionné est indispensable.

La possibilité de constituer une science ayant pour objet une certaine catégorie de phénomènes, en l'occurrence une science juridique portant sur les phénomènes juridiques, repose sur la capacité d'acquérir, dans ce domaine, une connaissance objective, c'est-à-dire une connaissance neutre, indépendante des caractéristiques psychologiques de l'observateur'. Par suite, il est nécessaire, d'une part, que l'objet des investigations soit effectivement saisissable objectivement et empiriquement et, d'autre part, que le chercheur dispose des moyens adéquats pour transmettre sans falsification et sans intrusion de la subjectivité les données recueillies, les analyses opérées et les conclusions déduites. En science juridique, lesdits moyens, sans s'y réduire, correspondent pour une large part aux concepts. Mais ils n'empêchent pas l'objectivité des juristes membres de la doctrine d'être qualifiée d' «illusion » ${ }^{2}$ et il est vrai que, s'ils ont une prétention scientifique, ils ne s'adonnent pas à proprement parler à une science ${ }^{3}$, cela peut difficilement être nié. Cette objectivité scientifique est un horizon dans la direction duquel il faut avancer tout en ne feignant pas d'ignorer qu'il est inatteignable. Les découvertes et le progrès scientifiques opérés dans le cadre de la science du droit ne revêtiront jamais les mêmes rectitude et exactitude que ceux opérés dans le cadre des sciences de la nature. Le chercheur, en science juridique et dans les sciences sociales et humaines plus généralement, adopte nécessairement un point de vue particulier ${ }^{4}$, y compris lorsqu'il s'efforce à la neutralité. Et John Locke, il y a déjà longtemps, de préciser qu'il n'est pas de concepts objectifs ${ }^{5}$. La scientificité des travaux de la doctrine juridique ne peut donc, quels que soient les efforts entrepris, rivaliser avec celle qui s'attache à la science physique ou à l'astronomie qui étudient des données naturelles, il n'est guère besoin de disserter plus longtemps pour en convenir. Ce qui importe et qui est l'objet des présents développements est que la scientificité de la «science » juridique qui s'appuie sur des concepts monosémiques est supérieure à celle de la «"science" » juridique qui emprunte la voie des concepts polysémiques.

${ }^{1}$ K. POPPER, La logique de la découverte scientifique (1959), Payot, 1973, p. 41.

${ }^{2}$ R. RICCI, «Le statut épistémologique des théories juridiques: essai de définition d'une pratique scientifique juridique, Dr. et société 2002, p. 156.

3 Ibid.

${ }^{4}$ K. POPPER, Conjectures et réfutations - La croissance du savoir scientifique, trad. M.-I. et M. B. de Launay, Payot (Lausanne), 1985, p. 79.

${ }^{5}$ J. LOCKE, Essai philosophique concernant l'entendement humain, 1735, L. I. 
Le «concept de concept ${ }^{1}$ doit être interrogé. Un concept est un outil linguistique (un mot ou une expression) servant à désigner abstraitement des objets « réels» - ce qui ne signifie pas «tangibles » ou «naturels» - . Participant d'un métalangage - un langage sur le langage - , les concepts permettent de condenser des informations, de généraliser des faits constatés par observation dans le monde empirique, de classer, différencier, ordonner, clarifier pour, in fine, comprendre puis expliquer le droit. Ils contribuent à surpasser le reproche formulé par Max Weber lorsqu'il regrettait la "prolixité des discours "intuitifs" [qui] masque seulement le fait que l'on est incapable de prendre ses distances par rapport à l'objet $»^{2}$. Le concept est ainsi une garantie contre le risque de journalisme juridique, soit le risque de se borner à la simple description de «faits normatifs », sans apport conceptuel et cognitif aucun. Il est donc au cœur de la structure épistémique du droit. Le concept ne sert pas uniquement à désigner un objet, sinon il serait appelé «notion » ${ }^{3}$; il permet de traduire une prise de position par rapport à celui-ci, si bien que, souvent, plusieurs concepts désignent un même objet - mais, normalement, plusieurs objets ne peuvent pas être désignés par un même concept - . Il n'est pas qu'une représentation verbale d'une ou plusieurs données brutes; il est une représentation orientée, faisant sens. Un concept, c'est une idée qui accompagne un objet; le langage devient alors plus qu'un moyen ${ }^{4}$.

Certains théoriciens du droit, à l'image de Duguit, ont entendu « éliminer tout concept du droit $»^{5}$ car ceux-ci rendraient les juristes raides et dogmatiques, car les représentations conceptuelles ne feraient souvent «qu'égarer la recherche en substituant les écarts désordonnés de la pensée à l'emprise seule féconde et sûre des réalités » ${ }^{6}$, car l' «expérience juridique immédiate » serait par trop « déformée et voilée par une épaisse croûte conceptuelle $»^{7}$. Les concepts sont néanmoins un outil important pour les législateurs qui, sans eux — et sans les catégories ${ }^{8}$-, ne

${ }^{1}$ F. COLONNA D'ISTRIA, «Le concept de concept juridique », RRJ-Cahiers de méthodologie juridique $2012, n^{\circ} 26$.

${ }^{2}$ M. Weber, L'Éthique protestante et l'esprit du capitalisme (1920), Pocket, coll. Agora, 1967, p. 23-24.

${ }^{3} C f$. W. Dross, «L'identité des concepts juridiques : quelles distinctions entre concept, notion, catégorie, qualification, principe ? », RRJ-Cahiers de méthodologie juridique 2012, $\mathrm{n}^{\circ} 26$.

${ }^{4}$ Par suite, le Vocabulaire de la philosophie d'André Lalande distingue : « $1^{\circ}$ Les concepts a priori ou purs », c'est-à-dire les concepts qui ne dérivent pas de l'expérience, et " $2^{\circ}$ Les concepts a posteriori ou empiriques » $\left(\mathrm{V}^{\circ}\right.$ «Concept », in A. LALANDE, Vocabulaire technique et critique de la philosophie, Puf, coll. Quadrige dicos poche, 2010). Il n'est pas lieu, ici, de distinguer de la sorte différents types de concepts. Simplement est-il possible d'observer que, par nature, les premiers sont plus que les seconds menacés par le danger de la polysémie.

${ }^{5}$ L. Duguit, Traité de droit constitutionnel - Tome premier : La règle de droit, le problème de l'État, $3^{\mathrm{e}}$ éd., De Boccard, 1927, p. 72.

${ }^{6}$ F. GÉNY, Science et technique en droit privé positif, t. III, Librairie du Recueil Sirey, 1921, p. 198.

${ }^{7}$ G. GURVITCH, L'expérience juridique et la philosophie pluraliste du droit, Pedone, 1935, p. 63.

${ }^{8}$ Cf. M. WALINE, «Empirisme et conceptualisme dans la méthode juridique - Faut-il tuer les catégories juridiques ? », in Mélanges Jean Dabin, t. I, Sirey, 1963, p. 363 s. 
produiraient qu'un fatras de règles hétéroclites, incohérentes, inapplicables, inaptes à régir les faits sociaux; alors qu'un réseau de concepts - et de catégories juridiques permet au droit positif d'adopter l'allure d'une organisation intellectuelle et, grâce à cela, d'atteindre ses objectifs pratiques ${ }^{1}$. Et ils sont également fort utiles aux juges, applicateurs du droit, en ce qu'ils permettent de passer du fait au droit, c'est-à-dire de qualifier juridiquement les faits ${ }^{2}$.

Contre ces dénégations des quelques rares contempteurs des concepts que furent Duguit ou Gurvitch, il faut croire que ceux-ci intéressent au premier chef les théoriciens du droit et, plus simplement, la doctrine et la science du $\mathrm{droit}^{3}$ seulement la doctrine, lorsqu'elle ne fait pas œuvre théorique, recourt-elle bien souvent à des concepts «prêts à l'emploi » trouvés dans les énoncés qui expriment les normes et institutions positives ${ }^{4}$ - On fait même de la proposition et du développement de concepts juridiques l'objet premier de toute théorie du droit, bien plutôt que la recherche d'une hypothétique vérité ou essence du droit ou que la formulation d'une indubitablement insatisfaisante définition du droit ${ }^{5}$. Bobbio expliquait, en ce sens, que la fonction de la théorie juridique est, notamment, de «fournir des instruments conceptuels unificateurs par-delà et au travers des dogmatiques juridiques construites à partir des divers droits positifs $»^{6}$. La doctrine publiciste, par exemple, comporte un grand nombre de concepts élaborés par les théoriciens du droit public, d' «État» à «souveraineté », en passant par « démocratie », «État unitaire », «État fédéral ${ }^{7}$ ou « régime parlementaire ${ }^{8}$. Certains de ces concepts sont ensuite repris par la Constitution ou par la loi. Et Olivier Beaud d'écrire que «ce n'est certainement pas le juge qui peut dire le dernier mot sur la notion de souveraineté qui est un concept doctrinal $»^{9}$. La théorie

${ }^{1}$ J.-L. BeRgEL, Théorie générale du droit, $5^{\mathrm{e}}$ éd., Dalloz, coll. Méthodes du droit, 2012, p. 225.

${ }^{2}$ O. CAYla, « La qualification ou la vérité du droit », Droits 1993, n 18, p. 3 s.

${ }^{3}$ Il n'est pas lieu, en ces lignes, de chercher à préciser la distinction entre « science juridique » et « doctrine juridique » car ce qui est en cause est la scientificité du discours tenu par les auteurs composant la doctrine. Sur ce point, $c f$. J. CHEVALLIER, « Doctrine juridique et science juridique », Dr. et société 2002, p. 103 s. ; E. PICARD, «Science du droit ou doctrine juridique », in Mélanges Rolland Drago, LGDJ, 1989, p. 12 s. Peut-être, si science du droit et doctrine entendent toutes deux produire de la connaissance sur le droit, seule la seconde cherche-t-elle également à participer de son élaboration, adoptant à cet instant non plus un point de vue extérieur et scientifique mais un point de vue intérieur au droit et intéressé. Ici, importe essentiellement la question de la connaissance du droit, si bien qu'il est permis de considérer que science du droit et doctrine se recouvrent largement.

${ }^{4}$ M. Troper, Pour une théorie juridique de l'État, Puf, coll. Léviathan, 1994, p. 188.

5 A. JEAMmaUd, « La règle de droit comme modèle », D. 1990, p. 199.

${ }^{6}$ N. BobBio, «Pour un lexique de théorie générale du droit», in Essais de théorie du droit, trad. Ch. Agostini, M. Guéret, LGDJ-Bruylant, coll. La pensée juridique, 1998, p. 63.

${ }^{7}$ Ch. EISENMANN, «Quelques problèmes de méthodologie des définitions et des classifications en science juridique », Arch. phil. droit 1966, p. 26.

${ }^{8}$ M. Troper, Pour une théorie juridique de l'État, Puf, coll. Léviathan, 1994, p. 188.

${ }^{9}$ O. BEAud, La puissance de l'État, Puf, coll. Léviathan, 1994, p. 12. 
juridique, qui peut elle-aussi être scientifique $^{1}$, se présente donc comme une «science constructive et conceptuelle $»^{2}$. Ainsi que le soulignait Kant, les concepts ont pour fonction de conférer aux raisonnements et aux développements du chercheur non une validité subjective, ne présentant d'intérêt que dans l'esprit du sujet pensant, mais une validité objective ${ }^{3}$, donc une validité scientifique ou, du moins, plus scientifique. La science appelle les concepts autant que les concepts appellent la science.

Cette dernière, a fortiori lorsqu'elle se rapproche des recherches théoriques, apparaît telle « une création humaine, et il devient désormais possible de considérer que son histoire est partie intégrante de l'histoire des idées ${ }^{4}$. Mais — et c'est là le cœur de la présente étude - les concepts, qui comptent parmi les principaux architectes de cette «science-création humaine », se doivent d'être monosémiques, c'est-à-dire de n'être toujours liés qu'à un et un seul signifié afin de ne pas laisser le lecteur ou l'auditeur dans une incertitude qui est ici jugée ascientifique. Or, «résultat[s] de l'effort de l'esprit en vue de saisir dans une représentation prédominante l'essence logique des choses $»^{5}$, ils portent nécessairement une grande part d'artificialité qui les fragilise. Ils sont fatalement «empreints d'un déterminisme conceptuel les rattachant à la justification d'un point de vue ${ }^{6}$, ce qui tend à éloigner les juristes d'une véritable pratique scientifique telle que l'envisage le rationalisme critique. Quels que soient les efforts fournis, la relative indétermination de la langue juridique, la certaine malléabilité de ses termes et la divergence potentielle entre ce qu'un juriste exprime et ce qu'un autre comprend ne sauraient s'évanouir totalement. Plaider pour l'utilisation de concepts monosémiques, ce n'est pas plaider de manière utopique pour une science juridique qui serait l'égale des sciences de la nature mais plaider pour une science juridique qui se rapproche du niveau maximum de scientificité auquel son particularisme ontologique donne droit. On considère souvent qu'il y a science lorsque sont possibles des vérités scientifiques semblables à celles qu'identifient les physiciens, les médecins ou les géographes; les vérités mises en exergue par les juristes ne seront jamais de pareilles vérités. S'il est vrai que " toute vérité est relative à une structure de l'esprit, à une psychologie ${ }^{7}$, cette assertion restera toujours plus pertinente à propos des « vérités juridiques » qu'à propos des « vérités naturelles ».

${ }^{1} C f$. B. BARRAUD, «L'échelle de juridicité : un outil pour mesurer le droit et fonder une théorie syncrétique », Arch. phil. droit 2013, p. 365 s.

${ }^{2}$ G. GuRvitch, L'expérience juridique et la philosophie pluraliste du droit, Pedone, 1935, p. 84.

${ }^{3}$ E. KANT, Prolégomènes à toute métaphysique future qui pourra se présenter comme science, Vrin, 1993, p. 66.

${ }^{4}$ K. POPPER, Conjectures et réfutations - La croissance du savoir scientifique, trad. M.-I. et M. B. de Launay, Payot (Lausanne), 1985, p. 272.

${ }^{5}$ F. GÉNY, Science et technique en droit privé positif, t. I, Librairie du Recueil Sirey, 1913, p. 148.

${ }^{6}$ R. RICCI, «Le statut épistémologique des théories juridiques: essai de définition d'une pratique scientifique juridique, Dr. et société 2002, p. 172.

${ }^{7}$ G. BACHELARD, La philosophie du non (1940), Puf, coll. Quadrige grands textes, 2005. 
L'utilisation des concepts s'est développée avec le nominalisme et Guillaume d'Occam, soit à partir du XIV $\mathrm{XI}^{\mathrm{e}}$ s., le plus important d'entre eux étant alors «homme ». Elle a été perfectionnée, plus tard, avec la méthode analytique défendant une position critique vis-à-vis du langage et, par conséquent, le recours à une expression précise et rigoureuse. Dans cette perspective, il s'agit moins de vérifier que les propositions correspondent à une réalité donnée que de rechercher si elles permettent directement et sans hésitation d'identifier l'objet visé par le concept, c'est-à-dire de s'assurer que ce dernier est monosémique. La monosémie est bien la première qualité à rechercher chez un concept puisque son propre doit être de faire sens. Il serait presque tentant d'avancer qu'il n'est de concept que monosémique, c'est-à-dire de faire de la monosémie un élément de la définition du concept ou, du moins, de la définition du concept juridique, d'autant plus que, même dans le cadre d'une science pré-paradigmatique, il devrait être possible de s'entendre sur certains concepts.

\section{B. La précision des concepts, exigence de la science juridique}

Certainement la poésie du droit est-elle une donnée incontestable, tant dans son pan positif que dans son pan doctrinal ${ }^{2}$; à tel point que le droit sans la poésie du droit serait sûrement un tout autre droit. Toutefois, en ces lignes, est plaidé pour l'extension de « la part de la science dans les activités des juristes $»^{3}$, spécialement par le truchement de l'emploi d'un arsenal conceptuel scientifique - et non poétique - , ce qu'il faut comprendre comme l'emploi d'un arsenal de concepts nonpolysémiques bien que des auteurs de plus en plus nombreux, relevant le caractère nécessairement ouvert du langage ordinaire (qui se confond en droit avec le langage scientifique), estiment qu'un concept, par nature, ne pourrait jamais être porteur d'un seul et unique sens. Si une science se conçoit comme un ensemble de connaissances raisonnées et coordonnées, assurément les activités des juristes méritent-elles d'être appelées «science». Seulement, pour pouvoir être ainsi raisonnées et coordonnées, est présentement soutenu que les connaissances juridiques doivent se voir exprimées au travers d'un langage simple et, surtout, précis, un langage incontestable, consensuellement accepté et largement compris.

Jean-Paul Sartre se montrait réservé quant à l'intérêt de la poésie car, disait-il, celle-ci, parce qu'elle joue avec les sons et la phonétique, prend le langage comme objet matériel, alors que le propre du langage est, avant toute autre chose, d'être un univers de significations chargé d'éclairer la vie humaine individuelle et collective. Partant, l'écrivain ne serait pas celui qui manie les sons - celui-là serait le musicien - mais celui qui manie les significations ${ }^{4}$. En matière d'écriture, et plus

\footnotetext{
${ }^{1}$ M. VILley, La formation de la pensée juridique moderne (1968), Puf, 2003, p. 46.

2 A. LAINGUI, « La poésie dans le droit », Arch. phil. droit 1996, p. 134 s.

${ }^{3}$ P. AMSELEK, «La part de la science dans les activités des juristes », D. 1997, p. 337 s.

${ }^{4}$ J.-P. SARTRE, Qu'est-ce que la littérature?, Gallimard, coll. Blanche, 1948.
} 
encore en matière d'écriture scientifique, le fond paraît être une nécessité quand la forme serait davantage un luxe, quoiqu'un luxe permettant au fond de « remonter à la surface » comme l'aurait dit — la citation est certainement apocryphe — Victor Hugo, ce qui n'est pas la moindre des fonctions, si bien qu'il n'est peut-être pas de fond sans forme et que, en définitive, le fond ne serait finalement rien d'autre que la forme. Il faut amplement relativiser la distinction de la matière et de la manière tant l'une et l'autre tendent à se rejoindre et même à se confondre. Reste que le poète - même s'il faut avancer, contre Sartre, qu'il existe « des poésies » plutôt que «la poésie»- serait davantage un musicien qu'un écrivain, tandis que le scientifique du droit devrait être un écrivain et seulement en de rares circonstances un poète-musicien - le plan en deux parties et la symétrie des intitulés tiennent plus de l'art et de la poésie que de l'expression scientifique - Et le scientifique du droit, plus que les autres écrivains, se doit d'utiliser un langage rigoureux, précisément fixé et strictement respecté au sein de la communauté scientifique.

Le langage juridique est, à un moment donné et dans un pays donné, la façon particulière dont la langue de ce pays est utilisée pour désigner ce qui relève du domaine du droit ${ }^{1}$. Ferdinand de Saussure, père de la linguistique moderne, définissait la langue comme « un système de signes exprimant des idées, [...] un système de pures valeurs que rien ne détermine en dehors de l'état momentané de ses termes ", lequel est le fait d'un consensus social, d' " une sorte de contrat passé entre les membres de la communauté ${ }^{2}$. Un individu ne saurait donc, seul, bâtir un langage. Celui-ci ne peut qu'être le fruit de l'action d'une communauté et, concernant le langage scientifique, le fruit de l'action d'une communauté scientifique. Et il en va de même des unités individuelles de la langue scientifique que sont, parmi d'autres, les concepts. Idéalement, il faudrait que chaque membre de ladite communauté linguistique ait "déposé dans [le] cerveau un dictionnaire identique $»^{3}$. En tout cas est-il vrai que la dimension collective du langage impose des contraintes pesantes à ses utilisateurs. Ainsi que le soulignait également Ludwig Wittgenstein, la signification d'un terme ou d'une expression n'est pas autre chose qu'un usage ; elle est donc un fait historique et sociologique ${ }^{4}$. Un concept ne prend sens qu'en situation, en fonction de sa réception cognitive par le lecteur ou auditeur. Le signifié d'un mot n'a pas de réalité autonome ; il est le résultat de l'interaction entre locuteur et interlocuteur ou entre écrivain et lecteur qui le produisent et le négocient en utilisant ce mot dans des circonstances déterminées. Il n'est donc pas suffisant de vouloir conférer une signification précise et unitaire à un concept pour que celui-ci possède effectivement une signification précise et unitaire. Cette dernière dépend autant — si ce n'est plus — des récepteurs ou destinataires du concept que de ses émetteurs. Or il peut être difficile de se faire une idée non-

${ }^{1}$ G. CORNU, « Linguistique juridique », in Dictionnaire de la culture juridique, op. cit., p. 952.

${ }^{2}$ F. DE SAUSSURE, Cours de linguistique générale (1916), Payot, 1979, p. 33.

${ }^{3}$ Ibid., p. 37.

${ }^{4}$ L. WitTGEnSTEIN, Recherches philosophiques (1953), Gallimard, coll. NRF - Bibliothèque de philosophie, 2005. 
ambigüe d'un concept dès lors que différents auteurs l'utilisent en y attachant des signifiés variables. Les concepts - qui sont à la fois les locutions verbales et leurs sens - risquent donc de se retrouver atteints par la polysémie, bien que l'intention de leurs «auteurs » soit de consacrer des concepts clairs et précis, dignes d'une discipline scientifique.

Par définition, un concept est une « représentation stable » d'un objet concret ou abstrait ${ }^{1}$. Surtout, la monovalence de ses concepts est une condition importante de scientificité d'une discipline. Certainement divers critères participent-ils de la scientificité de l'activité de la doctrine juridique et l'objectivité, l'empirisme, la transparence ou encore l'adoption d'un point de vue extérieur et descriptif ne sontils pas de peu de poids sous cet angle. Mais la monosémie - objet de la présente étude - devrait être prise en compte et, toutes choses égales par ailleurs, des enseignements appuyés sur des concepts monosémiques devraient être considérés comme autrement scientifiques que des enseignements appuyés sur des concepts polysémiques - comme des normes claires et précises semblent être, à l'aune de l'objectif d'accessibilité et d'intelligibilité des règles, plus juridiques que des normes ambivalentes laissant une large marge de manœuvre à leurs interprètes et, partant, étant source d'une insécurité dite «juridique ${ }^{2}-$ En un mot, les concepts monosémiques sont source de sécurité scientifique quand les concepts polysémiques sont source d'insécurité scientifique.

«Toute science, écrit-on, commence par l'apprentissage d'un langage ${ }^{3}$. Mais encore faut-il que ce langage soit à la fois complet et précis. Aussi, bien que la plupart des animaux sont dotés de la capacité de communiquer, seul l'humain est en mesure de s'adonner à des activités communicationnelles scientifiques. Marcel Proust regrettait qu' «on enferme les choses dans leurs noms » ${ }^{4}$; tandis que Montaigne souhaitait emprunter la voie d'un «parler ouvert $»^{5}$. Peut-être pareilles propositions sont-elles pertinentes pour qui entend faire œuvre philosophique ou, plus encore, poétique ; mais qui a pour ambition de faire œuvre scientifique devrait s'efforcer de recourir, dans la mesure de ce que les caractéristiques ontologiques de sa discipline lui permettent, à un langage fermé, donc à des expressions directement saisissables (ou presque, en impliquant un effort d'interprétation minimal). Dans l'idéal, tout scientifique ne devrait utiliser que les ressources fournies par un champ lexical scientifique, lequel serait identifié par une sémantique scientifique ${ }^{6}$. Déjà

${ }^{1} \mathrm{~V}^{\circ}$ « Concept », in Trésor de la langue française.

${ }^{2} C f$., sur la satisfaction à l'exigence de sécurité juridique comme critère de juridicité, B. BARRAUD, «L'échelle de juridicité : un outil pour mesurer le droit et fonder une théorie syncrétique », Arch. phil. droit 2013, p. 396-397.

${ }^{3}$ J.-M. CARBASSE, Introduction historique au droit, Puf, coll. Droit fondamental, 1998, p. 159.

${ }^{4}$ M. Proust, À la recherche du temps perdu - t. II : À l'ombre des jeunes filles en fleurs, Gallimard, coll. NRF, 1918.

${ }^{5}$ M. DE Montaigne, Les essais, 1588, L. III, chap. 1.

${ }^{6}$ Cf. A. TARSKI, « La construction d'une sémantique scientifique », in Logique, sémantique, métamathématique - 1923-1944, t. II, Armand Colin, 1974, p. 133 s. 
Confucius observait que, «quand les mots ne sont pas clairs, les jugements ne sont pas exacts, les œuvres ne prospèrent pas $»^{1}$. Cela peut tout à fait être étendu à la matière scientifique: "Quand les mots scientifiques ne sont pas clairs, les jugements ne sont pas exacts et les œuvres scientifiques ne prospèrent pas ». Avec les plus éminents penseurs de la science juridique, il convient donc d'affirmer qu' «un langage clair et précis est la première condition de toute étude scientifique $»^{2}$; que «c'est seulement parce que la tâche fondamentale de la science juridique consiste dans la construction d'un langage rigoureux, c'est-à-dire scientifique, qu'elle est une science au même titre que n'importe quelle autre science empirique ou formelle $»^{3}$. Plus le concept est monosémique, plus l'intermédiaire langagier est transparent et moins la différence entre le phénomène brut et l'objet étudié, soit le phénomène à travers le filtre des concepts, est grande.

L'inexactitude dans les définitions « naît et se perpétue souvent à la faveur de l'indétermination des mots qui [y] correspondent, de la pauvreté d'un langage qui ne fournit pas de termes différents pour distinguer les idées visées de leurs voisines $\gg$. Une définition pertinente et «efficace» scientifiquement ne doit pas contenir de termes nécessitant d'être à leur tour définis; elle doit pouvoir se passer de «définition de la définition $»^{5}$. Elle doit donc se composer de mots et expressions dont les sens sont l'objet d'un large consensus. A été souligné, par exemple à propos des recherches en droit administratif, combien se doter préalablement à l'étude d'outils sémantiques et conceptuels stables et clairs est impératif afin d'éviter l'incertitude du sens, laquelle a été «l'une des causes principales d'obscurité, de confusion, d'erreur même dans la question de la définition du droit administratif ${ }^{6}$. Par suite, la définition d'un concept ne peut être claire et précise que s'il est monosémique. Si le concept ne se rapporte pas à une définition mais à des définitions, chacune des sous-définitions peut bien être claire et précise, le concept n'en sera pas moins obscur et imprécis, quoique peut-être seulement hors contexte.

Par ailleurs, le discours du scientifique-juriste ne devrait pas, dans ce cadre, être porté par sa subjectivité mais par une ratio juris impersonnelle et objective - même si celle-ci est un objectif vers lequel il faut tendre tout en sachant qu'il ne saurait être parfaitement atteint — . Ledit scientifique-juriste devrait à tout prix s'efforcer de ne pas sombrer dans l' «opinion» sur laquelle aucune

${ }^{1}$ Cité par H. IsAR, «L'organisation structurale des pluralismes : contribution à une approche épistémologique de la pluridisciplinarité en sciences humaines et sociales », in O. BENOIST, H. ISAR, dir., Pluralisme, pluralismes, Presses universitaires d'Aix-Marseille, coll. Inter-normes, 2011, p. 228.

${ }^{2}$ R. CARRÉ DE MALBERG, Contribution à la théorie générale de l'État spécialement d'après les données fournies par le droit constitutionnel français, t. I, Librairie du Recueil Sirey, 1920, p. 259-260.

${ }^{3}$ N. BobBio, «La science du droit comme analyse du langage », in Essais de théorie du droit, trad. Ch. Agostini, M. Guéret, LGDJ-Bruylant, coll. La pensée juridique, 1998, p. 269.

${ }^{4}$ S. Romano, L'ordre juridique (1946), trad. L. François, P. Gothot, Dalloz, coll. Philosophie du droit, 1975 , p. 6.

${ }^{5}$ X. Magnon, Théorie(s) du droit, Ellipses, coll. Universités droit, 2008, p. 27.

${ }^{6}$ M. EISENMAN, Cours de droit administratif, t. I, LGDJ, 1982, p. 26. 
connaissance ne pourrait être fondée et qu'il faudrait, avec Gaston Bachelard, bannir de la pratique du chercheur ${ }^{1}$. Or s'exprimer au moyen de concepts monosémiques, reconnus, compris et acceptés par la communauté scientifique, est une première étape nécessaire sur le chemin de cette ratio juris, même si de nombreuses autres « règles du discours » doivent la compléter ${ }^{2}$. Et, au-delà de la seule problématique de la scientificité du propos doctrinal, on souligne que toute argumentation implique le respect de «présupposés logico-sémantiques» au nombre desquels figure l'obligation de « ne pas utiliser la même expression dans des sens différents $»^{3}$.

La polysémie est ainsi un ennemi de la science juridique telle que la comprend l'auteur de ces lignes. Néanmoins, selon les linguistes, la vocation d'un polysème est de revêtir, dans un texte, un seul de ses différents sens potentiels, s'actualisant en contexte. «L'ambiguïté, écrivait Gérard Cornu, n'est pas de l'essence de la polysémie; elle en est l'accident ${ }^{4}$. En la présente étude, toutefois, «polysémie d'un concept » est compris comme équivalent d' "ambiguité d'un concept »; c'està-dire qu'est concernée la polysémie des concepts hors et, surtout, en contexte. Partant, l'auteur de ces lignes, en retenant une signification de «polysémie » différente de celle adoptée par les linguistes — qui ne voient de polysémie que dans l'absolu —, participe de l'étonnant renforcement de la polysémie de « polysémie ». Les présents travaux étant destinés à un public de juristes et non à un public de linguistes, il n'est pas utile de discuter plus avant cette difficulté. Ce qui est en cause ici est la polysémie des concepts spécifiques à la doctrine juridique ; la polysémie de «polysémie » est une considération très secondaire.

Reste que l'invitation à la conservation et à la promotion de la monosémie dans le discours de la doctrine juridique pourrait recevoir un accueil chaleureux puisque le juriste est réputé intransigeant et rigide concernant le maniement du vocabulaire juridique et, au-delà, du langage commun : «Les étudiants se garderont de confondre deuxième et second, stipulation et disposition, résolution et résiliation, Conseil de 1'Europe et Conseil européen $»^{5}$. Cette exigence de rigueur et de précision s'impose certainement en matière de droit positif et Montesquieu notait à raison que « la loi ne doit pas contenir d'expressions vagues ${ }^{6}$. Si Stendhal disait lire chaque jour quelques articles du Code civil pour s'imprégner du style net et

${ }^{1}$ G. BACHELARD, La formation de l'esprit scientifique - Contribution à une psychanalyse de la connaissance objective, Vrin, 1938, p. 14.

${ }^{2}$ R. AleXY, «Idée et structure d'un système du droit rationnel », Arch. phil. droit 1988, p. 25 s.

${ }^{3}$ P. LIVET, «Argumentation et rhétorique juridique », in Dictionnaire de la culture juridique, op. cit., p. 85 .

${ }^{4}$ G. CORNU, « Linguistique juridique », in Dictionnaire de la culture juridique, op. cit., p. 954.

${ }^{5}$ P. DeUMIER, Introduction générale au droit, $2^{\mathrm{e}}$ éd., LGDJ, coll. Manuel, 2013, p. 12 ; également J.-

L. SOURIOUX, « Pour l'apprentissage du langage du droit », RTD civ. 1999, p. 343.

${ }^{6}$ Montesquieu, De l'esprit des lois, 1748, L. XXIV, chap. 16. 
lapidaire de ses rédacteurs ${ }^{1}$, et si le droit peut ainsi apparaître comme un empire du mot juste, on remarque et on critique aujourd'hui de plus en plus le fait que les législateurs tendent à adopter des dispositions excessivement larges et vagues s'apparentant à de véritables délégations normatives, cela afin de se défausser du soin d'édicter véritablement le droit, cette tâche incombant alors aux interprètesapplicateurs. Cette indétermination, souvent volontaire et même parfois assumée, peut s'expliquer par la nécessité d'éviter que les dispositifs en cause ne deviennent trop rapidement obsolètes. L'intérêt de recourir à des notions évolutives et flexibles apparaît, sous cet angle, patent. Ladite indétermination trouve aussi son origine dans la mauvaise maîtrise, par les législateurs, des enjeux et intérêts en cause, lesquels sont de plus en plus techniques à mesure que les sociétés et les environnements se complexifient. Et un nombre croissant de branches du droit positif sont touchées par les «notions fonctionnelles » et les «notions indéterminées ». De l'aveu même de certains membres du Conseil constitutionnel français, des formules au périmètre et à la portée incertains sont introduites dans la loi dans l'attente des commentaires de la doctrine et de l'application des juges, de sorte que « la boutade "j'attends de lire votre commentaire pour comprendre ce que j'ai décidé" n'est pas toujours une coquetterie $»^{2}$. De plus, le droit positif connaît depuis toujours les «standards» ou «notions-cadres », tels que «bon père de famille », "bonnes mœurs », "intérêt général », «ordre public» ou encore «équité naturelle ${ }^{3}$, qui permettent, moyennant l'appréciation nécessaire d'un juge, de déboucher sur une série infinie d'applications ${ }^{4}$. L'indétermination ne s'analyse alors pas comme un défaut mais bien comme une qualité puisqu'elle se comprend en tant qu' "élasticité » facteur d'adaptabilité et de complétude du droit. Sans ses multiples «notions critiques » ${ }^{5}$, nul doute que le droit positif serait excessivement incomplet.

En ces lignes, il s'agit d'envisager la problématique du «droit platonique ${ }^{6}$ telle qu'elle se présente au sein du versant doctrinal-scientifique du droit. Or celui-ci semble souffrir actuellement d'un mouvement similaire à celui que connaît le droit positif, allant de la précision vers l'imprécision. Mais la grande différence est que l'évolution en cause est cette fois involontaire et subie. Surtout, elle semble être

${ }^{1}$ Cité par A. LECA, La lyre de Thémis ou la poésie du droit, Presses universitaires d'Aix-Marseille, 2011, p. 60.

2 J. Pini, in «Débats et discussions », in M. Fatin-Rouge StÉFAnini, L. GAY, J. PinI, dir., Autour de la qualité des normes, Bruylant, coll. À la croisée des droits, 2010, p. 305.

${ }^{3}$ L'article $565 \mathrm{du}$ Code civil est ainsi un bon exemple : «Le droit d'accession [...] est entièrement subordonné aux principes de l'équité naturelle». En réalité, cela signifie que le pouvoir est délégué aux juges qui deviennent des sortes de «ministres d'équité ».

${ }^{4}$ Cf., par exemple, S. RIALS, Le juge administratif français et la technique du standard, LGDJ, coll. Bibliothèque de droit public, 1980

${ }^{5}$ Réf. à S. Rials, «Les standards, notions critiques du droit », in Ch. Perelman, R. VANDER Elst, dir., Les notions à contenu variable en droit, Bruylant, coll. Travaux du Centre national de recherches de logique, 1984, p. 40 s.

${ }^{6}$ R. LIBCHABER, «Qu'est-ce qu'une loi ?», RTD civ. 1999, p. 242. 
préjudiciable à la scientificité des études juridiques. Si les «notions à contenu variable » peuvent, lorsqu'elles s'attachent à des normes positives, présenter, en certaines circonstances, une réelle légitimité ${ }^{1}$, il ne paraît pas exister de circonstances qui leur permettent, en droit scientifique, de revêtir une semblable légitimité. Aussi note-t-on pertinemment que «la langue du droit [positif] est une langue non-scientifique, $[\ldots]$ un langage ordinaire. Les termes qu'elle emploie n'obéissent pas à la rigueur que l'on demande aux propositions scientifiques. [...] [Elle] a besoin de flou. La langue de la science du droit ne peut se satisfaire de ces imprécisions. [...] Elle doit clarifier son langage objet pour pouvoir en rendre compte $»^{2}$. À l'aune de ces explications, apparaît combien la problématique de la polysémie est effectivement «problématique » — au sens ordinaire du mot (qui est donc polysémique hors contexte) - en matière de doctrine et de science du droit alors qu'elle ne l'est que peu — et de manière fort différente - à l'égard du droit positif. Aussi convient-il, en cette étude, de s'inquiéter uniquement de l' «épidémie de polysémite » qui frappe les penseurs et commentateurs du droit. En droit positif, il ne s'agit pas nécessairement de «maladie de la norme ${ }^{3}$ - même si on a légitimement dénoncé 1' « usage abusif des notions confuses ${ }^{4}$ — et, d'ailleurs, il semble que plus une notion juridique occupe une position centrale au sein de l'ordre juridique, moins elle se voit précisément définie ${ }^{5}$.

Une qualité première à rechercher dans une notion, un concept ou une théorie juridique est son unité ; de son unité dépendent sa logique, sa cohérence, sa rigueur, son utilité et son accessibilité, donc sa légitimité scientifique. En somme, si le droit lui-même n'est pas toujours droit, on ne peut parler du droit qu'en parlant droit.

\section{Les concepts polysémiques et l'ascientificité du discours doctrinal contemporain}

Comme le soulignait Merleau-Ponty, quand il y a trop de sens, il n'y a plus de sens $^{6}$. L'essence n'est pas dans les sens mais dans le sens. S'il est vrai que « l'esprit de discernement est ce qu'il y a au monde de plus rare avec les diamants et les

\footnotetext{
${ }^{1}$ V. FORTIER, « La fonction normative des notions floues », RRJ 1991, p. $755 \mathrm{~s}$.

${ }^{2}$ É. Millard, Théorie générale du droit, Dalloz, coll. Connaissance du droit, 2006, p. 59-60.

${ }^{3}$ Réf. à C. BELOT, «La maladie de la norme - Rapport d'information fait au nom de la délégation aux collectivités territoriales et à la décentralisation sur les normes applicables aux collectivités territoriales », n 317 (2010-2011), déposé au Sénat le 16 févr. 2011.

${ }^{4}$ Ch. Perelman, «L'usage et l'abus des notions confuses », in Le raisonnable et le déraisonnable en droit - Au-delà du positivisme juridique, LGDJ, coll. Bibliothèque de philosophie du droit, 1984, p. 163.

${ }^{5}$ Ch. Atias, « Restaurer le droit du contrat », D. 1998, p. 137.

${ }^{6}$ M. Merleau-PonTy, Sens et non-sens (1948), Gallimard, coll. NRF - Bibliothèque de philosophie, 1996.
} 
perles ${ }^{1}$, posséder un arsenal conceptuel de qualité n'est toutefois pas un «luxe ». De la monosémie dépend l'aptitude à fournir des informations et des explications. Malheureusement, « en règle générale, seules des branches de la science, et pour un temps limité, acquièrent la forme d'un système de théories élaboré et logiquement bien construit $»^{2}$. Le besoin de s'appuyer sur des concepts monosémiques et d'écarter les concepts à tendance polysémique n'en doit pas moins se faire pressant, au contraire dès lors que cela contribue à conforter la logique théorique et à permettre l'acquisition et la transmission de connaissances objectives.

Deleuze et Guattari expliquaient que tout concept connaît trois stades successifs au cours de son développement : le moment de la formation, très long; le moment de la formulation, qui est une période de «nouage dans une cathédrale théorique »; et le moment de la «dégradation pédagogico-commerciale », au cours duquel le concept est vulgarisé et enseigné, «commercialisé » en quelque sorte ${ }^{3}$. Le temps de la formation correspond foncièrement à un temps de polysémie, tandis que celui du «nouage théorique » doit permettre le passage de la polysémie à la monosémie. Néanmoins, selon l'accueil et l'usage qui sont réservés au concept, la polysémie peut revenir lorsqu'il est l'objet d'une «dégradation pédagogicocommerciale». Mais la polysémie est essentiellement synonyme de «jeunesse conceptuelle » quand la monosémie coïncide avec la «maturité conceptuelle». En somme, s'il est des concepts polysémiques qui n'ont jamais été monosémiques, il n'est point de concepts monosémiques qui n'ont pas connu, antérieurement, la polysémie.

En droit, le vrai progrès scientifique est peut-être l'avancée conceptuelle, l'innovation cognitive par les concepts conçus comme des outils servant à la meilleure compréhension et explication du droit positif. La science du droit, de tout temps, se démarque par les nombreuses créations conceptuelles auxquelles procèdent les chercheurs qui la constituent. En conséquence, une certaine dose de polysémie est consubstantielle à la science du droit, parce qu'un concept n'est par nature jamais parfaitement monosémique et, surtout, parce que la doctrine juridique se nourrit d'évolutions conceptuelles. Or la sensible transition de la modernité à la postmodernité qui empreint la culture juridique contemporaine se caractérise, en particulier, par la prolifération des «notions à contenu variable » dont il vient d'être souligné combien, si elles peuvent revêtir quelque légitimité parmi le droit positif, elles ne le peuvent guère sous la loupe de la qualité des travaux doctrinaux. Face à

\footnotetext{
${ }^{1}$ J. DE LA BRUYÈRE, «Des jugements », in Les Caractères ou les Mœurs de ce siècle, 1688. André Gide écrivit, le 26 septembre 1926, ce commentaire qui ne manque pas de raisonner avec le propos tenu en la présente étude : «Je relis Les Caractères de La Bruyère. Si claire est l'eau de ces bassins, qu'il faut se pencher longtemps au-dessus pour en comprendre la profondeur » (A. GIDE, Journal : 1889-1939, Gallimard, coll. Bibliothèque de la Pléiade, 1951, p. 826).

${ }^{2}$ K. POPPER, La logique de la découverte scientifique (1959), Payot, 1973, p. 69.

${ }^{3}$ G. Deleuze, F. GuATtARI, Mille plateaux - Capitalisme et schizophrénie 2, Minuit, coll. Critique, 1980.
} 
ces mouvements intellectuels, quelques dictionnaires seraient assurément utiles ${ }^{1}$. Mais, plus encore, il peut sembler judicieux de ne pas employer de concepts polysémiques et de ne recourir qu'aux concepts monosémiques construits à l'ère de la modernité de la pensée juridique. Seulement, par définition, un «concept émergeant $»^{2}$, aussi longtemps qu'il demeure émergeant, ne peut qu'être mal défini et ambivalent. Dans le même temps, toute science construit son objet et, lorsque le réel qu'elle étudie procède à des évolutions et parfois même à des révolutions, elle se doit de reconstruire cet objet de façon adéquate afin de pouvoir rendre compte de ce réel nouveau ${ }^{3}$. En d'autres termes, si le droit positif moderne laisse place au droit positif postmoderne, alors la science moderne du droit devrait logiquement être subrogée par la science postmoderne du droit et la théorie moderne du droit par la théorie postmoderne du droit ${ }^{4}$.

Pareil processus ne peut se dérouler sans engendrer concomitamment une plus ou moins longue période d'incertitude et de malléabilité de l'outillage conceptuel qu'il est question de reconstruire partiellement. Or cette situation est sans doute préférable à celle qui résulterait du maintien à l'identique des concepts classiques en raison de la prévalence, parmi les juristes, d'une certaine paresse ou d'un certain conservatisme intellectuel ${ }^{5}$. En effet, dans ce dernier cas de figure, la scientificité serait impactée encore plus négativement que dans l'hypothèse de la construction de nouveaux concepts nécessairement polysémiques mais potentiellement capables de rallier autour d'eux les diverses règles et institutions engendrées par les mutations actuelles des phénomènes juridiques. Lorsqu'une science est une science sociale et que la société qui est son objet et qu'elle étudie mue, il lui revient de savoir opérer les changements pertinents afin de conserver une qualité épistémique au moins équivalente. Cela peut aller jusqu'à entraîner une « révolution scientifique », soit, au sens de Gaston Bachelard, un «bouleversement dans la structure de l'esprit » ${ }^{6}$. C'est un tel bouleversement que traduit l'idée de «postmodernité juridique », laquelle n'est pas le moins polysémique des concepts « postmodernes ».

Durkheim notait que, "s'il existe une science des sociétés, il faut bien s'attendre à ce qu'elle [...] nous fasse voir les choses autrement qu'elles n'apparaissent au vulgaire ; car l'objet de toute science est de faire des découvertes

\footnotetext{
${ }^{1}$ Par exemple, B. BARRAUD, «Dictionnaire du droit postmoderne », in Repenser la pyramide à l'ère des réseaux - Pour une conception pragmatique du droit, L'Harmattan, coll. Logiques juridiques, 2012, p. $305 \mathrm{~s}$.

${ }^{2}$ Réf. à E. LE DOLLEY, dir., Les concepts émergeants en droit des affaires, LGDJ-Lextenso, 2010.

${ }^{3}$ É. Millard, «Rendre compte du droit dans un contexte de globalisation », in J.-Y. ChÉROT, B. FRYDMAN, dir., La science du droit dans la globalisation, Bruylant, coll. Penser le droit, 2012, p. 51.

${ }^{4}$ Par exemple, A.-J. ARnAud, « De la régulation par le droit à l'heure de la globalisation », Dr. et Société 1997, p. 11 s.

${ }^{5}$ Ibid.

${ }^{6}$ G. BACHELARD, La philosophie du non (1940), Puf, coll. Quadrige grands textes, 2005.
} 
et toute découverte déconcerte plus ou moins les opinions reçues ${ }^{1}$. La découverte scientifique, dans les sciences sociales en général et dans la science juridique en particulier, semble être ainsi, d'une certaine façon et paradoxalement, une menace pour la scientificité de ces «sciences » en ce qu'elle est un facteur de polysémie. Mais sans doute est-ce une menace salvatrice. Si les changements qui affectent les caractéristiques du droit positif obligent à ériger de nouveaux concepts, ce qui a pour effet de marquer le contexte doctrinal actuel par une forte polysémie (A), la quête de scientificité des études juridiques conduit à plaider en faveur de la limitation de la tentation de faire œuvre doctrinale solitaire plutôt que solidaire (B). La polysémie et l'effacement de la modernité au profit de la postmodernité de la pensée juridique doivent être circonscrits à ce que l'évolution de l'objet-droit étudié impose. Or l'analyse de nombreux travaux récents permet de constater combien l'utilisation des concepts polysémiques est excessive.

\section{A. L'usage de concepts polysémiques, propre de la doctrine juridique contemporaine}

Selon Gérard Cornu, la polysémie serait une «marque essentielle du vocabulaire juridique », un «phénomène irréductible [car] le nombre des signifiés [y] est infiniment plus élevé que celui des signifiants $»^{2}$; et Christian Atias d'observer que « le langage juridique est volontiers caractérisé par sa précision, par sa technicité, $[\ldots]$ [mais, en réalité, il] est affecté d'une polysémie chronique » ${ }^{3}$. La polysémie a sans doute été chronique et palpable durant le siècle correspondant à la seconde modernité juridique $\left(\mathrm{le} \mathrm{XX}^{\mathrm{e}}\right)^{4}$ : le concept de « souveraineté », par exemple, semble avoir toujours été «pluriel, fluide, insaisissable et évolutif » 5 et il est qualifié de «mythe, slogan ou mot magique ${ }^{6}$, tandis que «contrat social » est un «concept encombré d'hésitations, au caractère controversable et indéfiniment malléable ${ }^{7}$. Mais la polysémie est surtout un caractère de l'état actuel de la doctrine, laquelle se voit contrainte d'entamer et d'approfondir la transition de la modernité à la

${ }^{1}$ É. DuRkheim, Les règles de la méthode sociologique, $2^{\mathrm{e}}$ éd. (1937), Puf, coll. Bibliothèque de philosophie contemporaine, 1973, p. VII.

${ }^{2}$ G. CORNu, «Linguistique juridique », in Dictionnaire de la culture juridique, op. cit., p. 954.

${ }^{3}$ Ch. ATIAS, Épistémologie juridique, Dalloz, coll. Précis, 2002, p. 190.

${ }^{4}$ J.-L. Autin, C. RiвOT et alii, Environnements - Les mots du droit et les incertitudes de la modernité Mélanges Jean-Philippe Colson, Presses universitaires de Grenoble, 2004.

${ }^{5} \mathrm{~K}$. BENYEKHLEF, Une possible histoire de la norme - Les normativités émergentes de la mondialisation, Thémis, 2008, p. 59.

${ }^{6} \mathrm{~L}$. BAL, Le mythe de la souveraineté en droit international - La souveraineté des États à l'épreuve des mutations de l'ordre juridique international, th., Université de Strasbourg, 2012, p. 19. L'auteur dénombre une dizaine de significations différentes de «souveraineté et souligne qu'il y a « autant de théories de la souveraineté que de disciplines » (ibid., p. 20).

${ }^{7}$ S. GOYARD-FABRE, « Contrat social (doctrines) », in Dictionnaire de la culture juridique, op. cit., p. 281. 
postmodernité de la pensée du droit, ce qui ne peut se faire sans quelques difficultés et, spécialement, sans une excroissance de concepts aux sens mal fixés. Il serait tout autant inconséquent d'ignorer les nouveaux phénomènes juridiques qui se donnent à voir au $\mathrm{XXI}^{\mathrm{e}}$ s. que de les commenter et analyser au moyen de l'arsenal conceptuel moderne. Aussi, nombreux sont les auteurs qui proposent et développent des concepts qui, il y a peu, étaient encore inconnus du vocabulaire juridique scientifique.

Le droit positif, évidemment, est premier dans l'avènement de la postmodernité juridique et cela se traduit par le recours à des notions polysémiques telles que « développement durable », «précarité », « dépendance », « conjugalité » et tant d'autres ${ }^{1}$. La polysémie parmi les règles et institutions positives est toutefois attestée depuis longtemps ${ }^{2}$; et a été souligné qu'en certaines circonstances elle peut être normale et même souhaitable ${ }^{3}$. Lorsque la société change, le droit doit être en capacité de la suivre et, à ces fins, les notions à contenu variable sont des instruments efficaces, spécialement lorsqu'ils sont maniés par les magistrats. Concernant la doctrine juridique, si la présence de quelques concepts polysémiques est naturelle et inévitable, car s'attacher à l'évolution d'un droit positif qui lui-même s'attache à l'évolution de la société et de son environnement oblige à édifier "dans l'urgence » de nouveaux concepts, il semble néanmoins qu'il soit, actuellement, fait un usage déraisonnable des termes et expressions plurivoques. Le début du $\mathrm{XXI}^{\mathrm{e}} \mathrm{s}$. ne peut qu'être, pour la doctrine et la science juridiques, " une période de transition paradigmatique par conséquent caractérisée par l'instabilité du sens » ${ }^{4}$, mais les juristes ne doivent pas voir en cela un prétexte pour ne pas, malgré tout, chercher à attacher à leur travaux le plus haut niveau de scientificité qu'il soit permis d'envisager dans le contexte théorico-épistémologique actuel. Par exemple, un intitulé dans lequel le sujet abordé est qualifié de «entre ombre et lumière » ${ }^{5}$ est certainement insignifiant — au sens étymologique du terme —, comme l'est la distinction de «ce dont il est question » et de «ce qui est en question ${ }^{6}{ }^{\text {ou celle de }}$ la «compréhension explicative du droit» et de l' «explication compréhensive du droit $»^{7}$.

${ }^{1}$ J.-L. BERGEL, « Le processus des concepts émergeants », in E. LE DOLLEY, dir., Les concepts émergeants en droit des affaires, LGDJ-Lextenso, 2010, p. 439 s.

${ }^{2}$ Par exemple, J. BeCQuART, Les mots à sens multiples en droit civil français - Contribution au perfectionnement du vocabulaire juridique, Puf, 1928.

${ }^{3}$ Par exemple, V. ForTIER, « La fonction normative des notions floues », RRJ 1991, p. 755 s.

${ }^{4}$ S. Lebel-Grenier, Pour un pluralisme juridique radical, th., Université McGill de Montréal, 2002, p. 2

${ }^{5} \mathrm{M}$. MEKKI, «La force normative des groupes d'intérêt : entre ombre et lumière », in C. THIBIERGE, dir., La force normative - Naissance d'un concept, LGDJ-Bruylant, 2009, p. 233 s.

${ }^{6}$ M. MeYer, Questionnement et historicité, Puf, 2000, p. 140 ; repris par Ch. ATIAS, «La question de droit », RTD civ. 2010, p. 249.

${ }^{7}$ M. LOISELLE, «L'analyse du discours de la doctrine juridique : l'articulation des perspectives interne et externe ", in CURAPP, Les méthodes au concret - Démarches, formes de l'expérience et terrains d'investigation en science politique, PUF, 2000, p. 188. 
D'aucuns conviennent que la théorie du droit est aujourd'hui plus qu'hier alors qu'elle devrait l'être moins qu'hier — « polyphonique ${ }^{1}$, ce qui explique sans doute, en partie, son certain insuccès parmi les facultés de droit. Déjà Bobbio soulignait «l'état de confusion linguistique dans lequel se trouve actuellement la théorie générale du droit dont la fonction devrait être de fournir des instruments conceptuels unificateurs $»^{2}$. Or cet état de confusion paraît s'être subitement dégradé à l'orée du changement de millénaire. Le "pluralisme sémantique des expressions ${ }^{3}$ empêche chaque jour un peu plus toute présentation et toute identification de qualité scientifique. Et si le $\mathrm{XX}^{\mathrm{e}}$ s. a eu son Gurvitch — qui d'ailleurs n'était pas juriste soit un auteur faisant preuve d'une excessive créativité lexicale - à tel point que Claude Lévi-Strauss disait : «Plus je lis Gurvitch, moins je le comprends » ${ }^{4}$, ce siècle était néanmoins caractérisé par une certaine stabilité des sens, spécialement après que Kelsen ${ }^{5}$, Hart ${ }^{6}$ et quelques autres grandes figures de la théorie juridique ont écrit.

Certains revendiquent encore une affiliation au courant jusnaturaliste; or " "nature" est susceptible de 17 sens et "droit" de 15, donc "droit naturel" doit comporter 255 significations distinctes ${ }^{7}$. La polysémie de «droit naturel » apparaît gargantuesque, mais elle n'est cependant ni significative de la postmodernité de la pensée juridique, ni significative de la modernité de la pensée juridique. En revanche, est ainsi significatif le fait qu'on relève, au sein du langage métajuridique, la prolifération des « faux-amis ${ }^{8}$ et des 《 mots à sens multiples ${ }^{9}$. Les auteurs convoquent de plus en plus fréquemment l'idée de "paradigme », terme très «à la mode » dans la doctrine juridique, alors qu'on souligne combien nul ne parait savoir ce qu'est un paradigme ${ }^{10}$, si ce n'est vaguement en en faisant une conception théorique dominante dans une communauté scientifique. Mais ce sont principalement certains concepts récents et d'usage devenu trop prématurément courant qui sont caractéristiques de l'appétence de la postmodernité de la pensée

${ }^{1}$ A. JeAmmaud, « La règle de droit comme modèle », D. 1990, p. 199.

${ }^{2}$ N. BobBio, «Pour un lexique de théorie générale du droit», in Essais de théorie du droit, trad. Ch. Agostini, M. Guéret, LGDJ-Bruylant, coll. La pensée juridique, 1998, p. 63.

${ }^{3}$ E. JouAnNet, « Du droit des gens au droit international », in Dictionnaire de la culture juridique, op. cit., p. 463.

${ }^{4}$ Cité par J. LE GofF, Georges Gurvitch - Le pluralisme créateur, Michalon, coll. Le bien commun, 2012.

${ }^{5}$ Notamment, H. KelSEN, Théorie pure du droit, $2^{\mathrm{e}}$ éd., trad. Ch. Eisenmann, Dalloz, 1962 ; H. KELSEN, Théorie générale des normes, trad. O. Beaud, Puf, coll. Léviathan, 1996.

${ }^{6}$ Notamment, H. L. A. HART, Le concept de droit, trad. M. van de Kerchove, Publications des Facultés universitaires Saint-Louis, 1994.

${ }^{7}$ A. SÉRIAUX, « Droit naturel », in Dictionnaire de la culture juridique, op. cit., p. 508.

${ }^{8}$ B. BEIGNIER, « Droits (classification) », in Dictionnaire de la culture juridique, op. cit., p. 536.

${ }^{9}$ C. Puigelier, « Mots à sens multiples et règle de droit », Arch. phil. droit 2004, p. 381.

${ }^{10}$ M. MEYER, Découverte et justification en science - Kantisme, néo-positivisme et problématologie, Klincksieck, 1979, p. 321. 
juridique pour la polysémie. Tout d'abord, le concept de " postmodernité juridique » ou de «postmodernité de la pensée juridique » est lui-même parfaitement incertain et amphigourique ${ }^{1}$ — on fait, parfois, de Rousseau et Durkheim des penseurs postmodernes du droit ${ }^{2}$ - Et cela vaut peut-être à l'égard de la plupart des concepts comportant le préfixe «post- ${ }^{3}$. Avant de songer à penser la postmodernité ${ }^{4}$, il faudrait déjà avoir pensé la modernité ${ }^{5}$. Tandis que d'aucuns font de la globalisation la source première du développement d'un «droit moderne ${ }^{6}$ — et non « postmoderne »-, un auteur voit dans l' « instauration d'un contrôle effectif de constitutionnalité des lois » l'indice le plus significatif de l'avènement du droit postmoderne ${ }^{7}$. En exagérant quelque peu le trait, il semble qu'il y ait autant de postmodernités juridiques que de penseurs de la postmodernité juridique mais aussi, quoique dans une moindre mesure, autant de modernités juridiques que de penseurs de la modernité juridique.

Parmi les nombreux concepts polysémiques qui sont la marque de la pensée juridique au début du $\mathrm{XXI}^{\mathrm{e}}$ s. (2), « gouvernance» et « régulation » pourraient bien être les plus symptomatiques (1).

\section{1. «Gouvernance » et « régulation »}

Doit être tout d'abord précisé que, si le propos tenu en la présente étude est foncièrement critique, cette critique est adressée, en premier lieu, à l'auteur de ces lignes lui-même, celui-ci ne considérant en aucun instant qu'il échappe à la tendance à la polysémie décrite et combattue. Par suite, tirer des exemples de ses propres écrits ne serait pas représentatif, alors que ne pas illustrer les développements par quelques cas concrets serait inconvenant scientifiquement. Il faut donc rechercher les témoignages de la polysémie en cause parmi les œuvres doctrinales extérieures à l'auteur.

\footnotetext{
${ }^{1}$ Par exemple, B. Frydman, «Le droit, de la modernité à la postmodernité », in Réseaux 2000, $\mathrm{n}^{\circ} 88$, p. 67 s. ; B. DE SousA SANTOS, «Droit: une carte de lecture déformée - Pour une conception postmoderne du droit », Dr. et société 1988, p. 380 ; A.-J. ARNAUD, « Du jeu fini au jeu ouvert - Réflexions additionnelles sur le droit post-moderne », Dr. et société 1991, p. 43 s.

2 A. CARTY, «Du Postmodernisme en théorie et en sociologie du droit, ou Rousseau et Durkheim lus par Baudrillard », Dr. et société 1989, p. 371 s.

${ }^{3}$ Par exemple, J. Chevallier, L'État post-moderne, $3^{\mathrm{e}}$ éd., LGDJ, coll. Droit et société, 2008 ; L. RoubAN, «Les paradoxes de l'État postmoderne », Cités 2004, n 18, p. 11 s. ; R. DRAÏ, L'État purgatoire - La tentation postdémocratique, Michalon, 2005.

${ }^{4}$ Par exemple, F. OsT, « Jupiter, Hercule ou Hermès : quel modèle pour un droit postmoderne ? », Journal des procès $1990, \mathrm{n}^{\circ} 179-180$.

${ }^{5}$ Réf. à B. LATOUR, Nous n'avons jamais été modernes - Essai d'anthropologie symétrique, La découverte, 1997 ; $c f$. également Ch. TAYLOR, « Critique de la post-modernité », Société 1998, n 18-19.

${ }^{6}$ A. GidDEns, Les conséquences de la modernité, L'Harmattan, trad. O. Meyer, 1994, p. 69.

${ }^{7}$ B. FrYDMAN, « Le droit, de la modernité à la postmodernité », Réseaux 2000, n 88, p. 69.
} 
S'il fallait établir un palmarès des concepts au départ de deux critères qui seraient, d'une part, l'importance quantitative de l'usage par la doctrine juridique contemporaine et, d'autre part, le niveau qualitatif de polysémie, certainement « gouvernance » arriverait-il en tête, suivi de près par « régulation », ce dernier étant plus communément usité mais moins fondamentalement ambivalent que « gouvernance ». Nombre d'auteurs expliquent que les mutations qui imprègnent les phénomènes juridiques à l'ère des pluralismes et de la globalisation «s'accompagn[ent] de deux transformations majeures dans l'univers juridicopolitique : les passages de la règlementation à la régulation et du gouvernement à la gouvernance $»^{1}$. Il est permis de s'interroger quant à la pertinence de l'usage de ces termes, spécialement de «gouvernance $»^{2}$. Ce dernier concept est l'objet de débats quant à sa signification, sa définition, ses origines et ses conséquences, toutes étant incertaines. On relève que qui parle de «gouvernance» tient nécessairement un propos confus et ambigu ${ }^{3}$. Il peut s'agir tant de "gouvernance locale» que de «gouvernance urbaine», de «gouvernance d'entreprise», de «gouvernance de l'emploi », de «gouvernance mondiale», de «gouvernance des régions européennes » ou de «gouvernance multi-niveaux ${ }^{4}$; et la liste n'est nullement exhaustive $^{5}$, les diverses définitions de la gouvernance «s'échelonn[ant] de l'utopie à l'autorégulation ${ }^{6}$. L'idée est tellement «à la mode» qu'on a songé à ériger la «bonne gouvernance » en nouvelle loi du service public ${ }^{7}$. Tantôt dans le droit et tantôt hors du droit, tantôt avec le droit et tantôt contre le droit, pour l'heure, « gouvernance » est un concept à l'itinéraire par trop incertain, si bien qu'un auteur parle d' «itinéraires » ${ }^{8}$, traduisant ainsi la multiplicité des signifiés cachés derrière ce signifiant. Y compris en contexte, la gouvernance veut tout dire et, partant, ne veut rien dire. Alors que les dictionnaires de la langue française la définissent en une

${ }^{1}$ F. Ost, M. VAn DE Kerchove, De la pyramide au réseau ? - Pour une théorie dialectique du droit, Publications des Facultés universitaires Saint-Louis, 2002, p. 63.

${ }^{2}$ C. Mahieu, K. Volter, «Utilisation du concept de "gouvernance": Quelle(s) justification(s) ?», in G. Demuinnck, P. VerCAUTEREN, dir, L'État face à la globalisation économique - Quelles formes de gouvernance?, Sandre, 2009, p. 155 s.

${ }^{3}$ C. BARON, « La gouvernance : débats autour d'un concept polysémique », Dr. et société 2003, p. 330.

${ }^{4}$ Ibid.

${ }^{5}$ Également, par exemple, J. N. RoseneAu, E.-O. CZEMPIEL, dir., Governance without Government: Order and Change in World Politics, Cambridge University Press, 1992 ; J. LECA, « La gouvernance de la France sous la Cinquième République », in Mélanges Jean-Louis Quermonne, Presses de Sciences Po, 1996 ; R. DraÏ, «La gouvernance négative», Cités 2004, n 18, p. 87 s. ; O. PAYE, «Pour la gouvernance comme problématique générale de science politique », in G. DEMUIJNCK, P. VERCAUTEREN, dir, L'État face à la globalisation économique - Quelles formes de gouvernance?, Sandre, 2009, p. $129 \mathrm{~s}$.

${ }^{6}$ D. BourCIER, «Comment s'accorder sur les normes ? Le Droit et la Gouvernance face à Internet », Lex electronica 2006, vol. 10, $\mathrm{n}^{\circ} 3$, p. 9.

${ }^{7}$ J. Morand-Deviller, Droit administratif, 13 éd., LGDJ, coll. Cours, 2013, p. 474.

${ }^{8}$ Réf. à A. KAZANCIGIL, « La gouvernance : itinéraires d'un concept », in Mélanges Guy Hermet, Karthala, 2002, p. 121 s. 
demi-ligne ( « obéir au gouvernail, se (laisser) diriger $»^{1}$ ), assurément est-il judicieux d'utiliser d'autres mots et expressions afin d'exprimer la pensée en cause, la concision perdue étant largement compensée par la précision gagnée.

Quant au concept de « régulation », dont le droit de cité au sein de la doctrine juridique est allègrement affirmé, on note qu'il revêt un "caractère éminemment polysémique $»^{2}$, qu'il est « lourd d'ambiguités » ${ }^{3}$, qu'il correspond à des signifiés «divers voire opposés ${ }^{4}$ et qu'il n'est à utiliser que dans un contexte extrascientifique $^{5}$. On a pu dénombrer «cinq ou six acceptions» de « régulation ${ }^{6}$. Sans doute $\mathrm{y}$ en a-t-il désormais davantage; mais encore faudrait-il séparer les significations principales des significations accessoires ou marginales, c'est-à-dire envisager une polysémie qualitative et non seulement quantitative. Pour certains, rien dans le monde juridique n'est régulation car la régulation en droit n'existe pas, est ontologiquement inconcevable et, donc, est un «non-concept»; tandis que, pour d'autres qui adoptent une position inverse et symétrique à la suite de Carbonnier qui voyait dans « réguler » un «synonyme de "réglementer" » ${ }^{7}$, tout le droit s'apparente à de la régulation ${ }^{8}$; et on fait $\mathrm{du}$ «droit de la régulation » une pleine branche du droit ${ }^{9}$. Ainsi le terme « régulation » semble-t-il ne présenter qu'un faible intérêt pour le langage juridico-scientifico-doctrinal. «Le succès nouveau, fait-on valoir, que rencontre le vocable de "régulation" joue plutôt contre lui, car, à constater son usage à tout propos, on est tenté d'y voir un effet de mode, un procédé d'annonce [...], une astuce d'image pour masquer la misère d'un fourre-tout » ${ }^{10}$. Et de déplorer " l'état des réflexions doctrinales en France, qui d'ailleurs, curieusement, ont surtout été le fait à ce jour des auteurs privatistes alors qu'il s'agit fondamentalement d'une notion de droit public $\gg 11$.

${ }^{1} \mathrm{~V}^{\circ}$ « Gouvernance », in Trésor de la langue française.

${ }^{2}$ B. DU MARAIS, «Régulation de l'internet: des faux-semblants au retour à la réalité », RFAP 2004, $\mathrm{n}^{\circ} 109$, p. 83 .

${ }^{3}$ A.-J. ARNAUD, Critique de la raison juridique - 2. Gouvernants sans frontières - Entre mondialisation et post-mondialisation, LGDJ, coll. Droit et société, 2003, p. 93.

${ }^{4}$ Ibid.

${ }^{5}$ G. TIMSIT, « La régulation : la notion et le phénomène », RFAP 2004, n 109, p. 5.

${ }^{6}$ A. JEAMMAUD, «Introduction à la sémantique de la régulation juridique - Des concepts en jeu », in J. Clam, G. MARTIN, dir., Les transformations de la régulation juridique, LGDJ, coll. Droit et société, 1998, p. 47 s.

${ }^{7}$ J. CARbonnier, Droit civil - Introduction, $27^{\mathrm{e}}$ éd., Puf, coll. Thémis droit privé, 2002, p. 38.

${ }^{8}$ G. Timsit, « Normativité et régulation », Cah. Cons. const. 2007, n 21.

${ }^{9}$ G. Marcou, F. Moderne, dir., Droit de la régulation, service public et intégration régionale, L'Harmattan, coll. Logiques juridiques, 2005 ; M.-A. Frison-RocHE, « Définition du droit de la régulation économique », in M.-A. FRISON-Roche, dir., Les régulations économiques - Légitimité et efficacité, Presses de Sciences Po-Dalloz, coll. Droit et économie de la régulation, 2004.

${ }^{10}$ M.-A. FRISON-ROCHE, « Le droit de la régulation », D. 2001, p. 610.

${ }^{11}$ Ibid. 
Bien sûr, « dérégulation », autre notion à succès, correspond au moins autant à un signifié inaccessible que «régulation»; et il en va pareillement des sousconcepts dérivés que sont « régulation traditionnelle » ${ }^{1}$, « régulation spontanée », « autorégulation », « corégulation », « régulation avec le marché », « régulation par le marché » et « régulation hiérarchique $»^{2}$. C'est peu dire, donc, que le concept de régulation connaît «quelques usages ${ }^{3}$; et Jacques Chevallier peut écrire que celuici «apparaît comme un concept flou, polysémique, utilisé dans des sens extrêmement variés, voire passablement contradictoires ${ }^{4}$. Qu'on lui adjoigne un adjectif polysémisant tel que «flexible » ${ }^{5}$ et le caractère aventureux de son usage ne pourra qu'atteindre des sommets.

Il est remarquable que les notions de « régulation » et de «gouvernance »sont aussi souvent radicalement opposées que comprises comme synonymes ${ }^{6}$. Sous-titrer un article $^{7}$ ou une thèse ${ }^{8}$ « [sujet $\left.\lambda\right]$ : entre régulation et gouvernance » revient peu ou prou, tout bien considéré, à ne pas sous-titrer ledit article ou ladite thèse. Les concepts en cause, qui conquièrent sans cesse de nouveaux champs ${ }^{9}$, sont devenus, en outre, d'usage courant dans différentes disciplines, très au-delà de la seule science juridique, ce qui contribue grandement à les rendre polysémiques hors contexte. Aussi convient-il de séparer « régulation juridique » de « régulation ${ }^{10}$ et « gouvernance juridique » de « gouvernance » — comme un juriste se doit de parler de «normes juridiques » et non simplement de «normes»-. Ces concepts, parce que devenus très familiers des juristes, constituent un langage familier bien plus qu'un langage scientifique. Écrire que «la régulation est une nouvelle approche de la gouvernance » ${ }^{11}$ n'a ni plus ni moins de sens qu'écrire que « la gouvernance est une nouvelle approche de la régulation ».

${ }^{1}$ M. CROZIER, « La crise des régulations traditionnelles », in H. MENDRAS, dir., La sagesse et le désordre, Gallimard, 1980, p. 375 s.

${ }^{2}$ B. Du MARAIS, Droit public de la régulation économique, Presse de Sciences Po-Dalloz, 2004, p. 485 s.

${ }^{3}$ Réf. à J. Chevallier, «De quelques usages du concept de régulation », in M. Miaille, dir., La régulation, entre droit et politique, L'Harmattan, 1995, p. $71 \mathrm{~s}$.

${ }^{4}$ J. CHeVAlLIER, « La régulation juridique en question », Dr. et société 2001, p. 830.

${ }^{5}$ Réf. à J.-G. PADIOLEAU, «Les régulations flexibles», in Fondation IDATE, Piloter la société de l'information, Hermès, 2000.

${ }^{6}$ Par exemple, A.-J. ARnAud, Critique de la raison juridique - 2. Gouvernants sans frontières - Entre mondialisation et post-mondialisation, LGDJ, coll. Droit et société, 2003, p. 93.

${ }^{7}$ N. DREYFUS, «L'Icann entre régulation et gouvernance », RLDI 2012, n 81, p. $119 \mathrm{~s}$.

${ }^{8}$ V. Trovatello, L'infrastructure d'internet - Entre régulation et gouvernance, th., Université Paul Cézanne - Aix-Marseille III, 2009.

${ }^{9}$ M.-A. FRISON-Roche, « Les nouveaux champs de la régulation », RFAP 2004, n 109, p. 53 s.

${ }^{10}$ Par exemple, G. MARCOU, « La notion juridique de régulation », AJDA 2006, p. 347 s.

${ }^{11}$ V. Trovatello, L'infrastructure d'internet - Entre régulation et gouvernance, th., Université Paul Cézanne - Aix-Marseille III, 2009, p. 29. 
La polysémie frappant la doctrine juridique contemporaine est loin de se réduire à ces deux exemples que sont «régulation» et «gouvernance». La pathologie épistémologique se manifeste par divers autres symptômes.

\section{Autres illustrations}

Si «gouvernance » et «régulation" sont aujourd'hui des concepts très couramment utilisés par la doctrine juridique, ils ne sont aucunement les uniques manifestations de la polysémie qui frappe celle-ci. D'autres symptômes peuvent être identifiés, comme la tendance croissante à recourir à des affirmations porteuses de trop peu de sens ${ }^{1}$ ou à des citations tout aussi peu porteuses de sens telles que celle empruntée à Thomas d'Aquin selon laquelle «c'est l'un qui, de soi, est la cause de l'un; le multiple n'est cause de l'un qu'accidentellement selon que lui-même est un $»^{2}$. «L'un se puise dans le divers et la diversité trouve son sens dans l'unité $»^{3}$ est un beau slogan mais pas une affirmation scientifique, pas une assertion rigoureuse et précise.

Surtout, de multiples autres concepts sont actuellement mis sur le devant de la scène doctrinale alors que leurs sens sont exagérément incertains. Or leur succès, qui fait que de plus en plus nombreuses sont les études à les évoquer, est une barrière à leur « conceptualisation » — c'est-à-dire à leur précision — qui ne peut être que le fait d'une école ou, du moins, d'un ensemble d'auteurs liés par une harmonie cognitive. Que ces concepts soient à la fois émergents et dans le «commerce » doctrinal, pour reprendre le mot de Deleuze et Guattari, les empêche de trouver un terreau serein où se développer et s'épanouir scientifiquement parlant; un peu à l'image d'un tableau qui ne serait pas peint par l'artiste puis divulgué et exposé mais exposé $a b$ initio, en tant que toile quasi-vierge, pour être ensuite composé petit-àpetit, par touches successives, par le public ; nul doute que l'œuvre du maître serait autrement unitaire et cohérente que cette dernière.

Ainsi en va-t-il du «paradigme de la complexité » ${ }^{4}$ et des concepts de « source du droit », que déjà Kelsen qualifiait d' «inutilisable» car trop «étendu et

\footnotetext{
${ }^{1}$ Tel est le cas de « l'exigence d'efficacité du droit impose un rapprochement entre l'ordre et le désordre » (J. CATTAN, Le droit et les communications électroniques, th., Université d'Aix-Marseille, 2012, p. 16) tant il est difficile de saisir à quoi pourrait ressembler le visage de ce droit efficace à la fois ordonné et désordonné.
}

${ }^{2}$ Thomas D’Aquin, Somme théologique, XIII ${ }^{\text {e }}$ s., Ia, q. 11, art. 3 (cité par A. SÉRIAux, Le Droit - Une introduction, Ellipses, 1997, p. 30).

${ }^{3}$ A. SÉRIAUX, Le Droit - Une introduction, Ellipses, 1997, p. 60.

${ }^{4}$ Par exemple, S. Diebolt, «La complexité comme paradigme pour concevoir une régulation juridique adéquate », Dr. et société 2000, p. 501 s.; S. DieBOLT, Le droit en mouvement - Éléments pour une compréhension constructiviste des transformations complexes des systèmes juridiques, th., Université Paris X-Nanterre La Défense, 2000. 
imprécis $»^{1}$, de « réseau »- un « sac à métaphores $»^{2}$ «encombré de sens ${ }^{3}, \mathrm{y}$ compris en théorie du droit ${ }^{4}$ —, de «société civile», expression assimilée à un «terrain vague ${ }^{5}$ et à un « gadget $»^{6}$, et de « puissance », à propos duquel Raymond Aron soulignait que «peu de concepts ont été aussi couramment employés et sont aussi équivoques ${ }^{7}$. «Droit mou » et «droit souple », bien qu'ils ressemblent fort à des contradictio in adjecto, sont eux aussi régulièrement empruntés par les commentateurs et penseurs pour désigner les avis de la Cour de cassation autant que les recommandations de la Commission des clauses abusives, les directives administratives ou même certaines lois. D'ailleurs, la «théorie des sources du droit»se voit «problématiquement»- à tous les sens du terme - divisée en « sources du droit» et «sources du droit mou ${ }^{8}$. Droits «souple» et «mou »sont tout à fait emblématiques puisqu'ils consistent en des concepts qui eux-mêmes peuvent être qualifiés de «souples » et «mous» tant ils se révèlent vagues et indéfinis. Un auteur assimile le «droit souple » à une «notion mystérieuse » ${ }^{9}$ qui «recouvre des réalités on ne peut plus hétérogènes [...] qui rendent malaisé son approfondissement conceptuel ${ }^{10}$. Si le programme de la postmodernité de la pensée juridique est réellement d'interroger «le mou, le doux et le flou du droit ${ }^{11}$, alors il faut croire que la culture juridique gagnera en philosophie ce qu'elle perdra en scientificité ; mais il n'est pas certain qu'il faille s'en réjouir car, dans l'antre des facultés de droit, la science du droit paraît primer largement sur la philosophie du droit, bien que l'intérêt intellectuel de cette dernière soit incontestable. Il est vrai que le droit est généralement réduit à une technique appliquée et concrète quand la

${ }^{1}$ H. KELSEN, Théorie pure du droit, $2^{\mathrm{e}}$ éd., trad. Ch. Eisenmann, Dalloz, 1962, p. 134 ; également P. AMSELEK, « Brèves réflexions sur la notion de sources du droit », Arch. phil. droit 1982, p. 254.

${ }^{2}$ P. Musso, Télécommunications et philosophie des réseaux - La postérité paradoxale de Saint-Simon, Puf, 1997, p. 36.

${ }^{3}$ H. BAKIS, Les réseaux et leurs enjeux sociaux, Puf, 1993, p. 8.

${ }^{4}$ Réf. à F. OST, M. VAN DE KERChOVE, De la pyramide au réseau ? - Pour une théorie dialectique du droit, Publications des Facultés universitaires Saint-Louis, 2002.

${ }^{5}$ R. DRAÏ, L'État purgatoire - La tentation postdémocratique, Michalon, 2005, p. 100.

${ }^{6}$ D. LOSCHAK, «La société civile : du concept au gadget», in CURAPP, La société civile, Puf, 1986, p. $43 \mathrm{~s}$.

${ }^{7}$ R. Aron, Paix et Guerre entre les nations, Calmann-Lévy, 1962, p. 58.

${ }^{8}$ Y. CARtuyvels, H. Dumont, Ph. GÉrard, I. Hachez, F. Ost, M. van DE KerChove, dir., Les sources du droit revisitées - Volume 4 : Théorie des sources du droit, Publications de 1'Université Saint-LouisAnthémis, 2013.

${ }^{9}$ C. ThiBIERGE, « Le droit souple - Réflexion sur les textures du droit », RTD civ. 2003, p. 599.

${ }^{10}$ C. ThiBIERGE, « Rapport de synthèse », in Association Henri Capitant, Le droit souple, Dalloz, coll. Thèmes et commentaires, p. 144.

${ }^{11}$ M. Delmas-Marty, «Le mou, le doux et le flou sont-ils des garde-fous de nos sociétés dites postmodernes ? », in J. ClAM, G. MARTIN, dir., Les transformations de la régulation juridique, LGDJ, coll. Droit et société, 1998, p. 209. 
philosophie est souvent perçue comme par trop évasive en même temps que spéculative.

À la barre des accusés de polysémie, pourrait encore être cité le concept de «droit global», caractéristique autant de la postmodernité de la pensée juridique que des ambiguïtés qui l'imprègnent. Ledit concept est dénoncé comme étant une «idée théoriquement inutile ${ }^{1}$, un «Objet Juridique Non Identifié » ${ }^{2}$, un «motvalise attrape-tout [...] épouvantablement confus » et même une « faute de goût $»^{3}$. Également, «système » aurait toute sa place dans la liste des concepts plurivoques, lui dont « la générosité dans l'emploi par les juristes n'a d'égale que la disparité des significations qu'ils lui attribuent ${ }^{4}$. On présente volontiers le droit tel un système, mais cela ne permet guère d'accéder à son ontologie, même en partie, tant sont alors visées «les réalités empiriques les plus diverses et les plus hétérogènes ${ }^{5}$. «Système ouvert », « système fermé », « système de normes », « système social » ${ }^{6}$, 《système autopoḯtique ${ }^{7}$ et " grands systèmes de droit contemporains ${ }^{8}$ n'ont que peu de choses en commun. Et «pluralisme juridique» est assurément un autre concept extraordinairement polysémique. Entre celui décrit par Santi Romano ${ }^{9}$ et celui qu'envisagent les sociologues et anthropologues du droit, il y a un fossé, si ce n'est un gouffre, sémantique. Un auteur estime que le pluralisme juridique repose sur de l' « à-peu-près » ${ }^{10}$ et qu'il n'a « rien à dire » ${ }^{11}$, peut-être parce que, justement, il en dit trop, lui qui fait partie de «ces concepts sur-sollicités $»^{12}$. Et d'ajouter qu' «il y a dans [cette expression] quelque-chose d'inachevé, d'inaccompli : nous

${ }^{1}$ F. Rouvière, «La globalisation du droit: une idée théoriquement inutile », in J.-Y. CHÉROT, B. FRYDMAN, dir., La science du droit dans la globalisation, Bruylant, coll. Penser le droit, 2012, p. $115 \mathrm{~s}$.

${ }^{2}$ B. Frydman, «Comment penser le droit global ? », in J.-Y. ChÉRot, B. Frydman, dir., La science du droit dans la globalisation, Bruylant, coll. Penser le droit, 2012, p. 17.

${ }^{3}$ M. XIfARAS, «Après les Théories Générales de l'État : le droit global ? », Jus Politicum 2012, n 8, p. 4. L'auteur décrit ainsi le préjugé de certains auteurs mais, pour sa part, il entend défendre l'utilité du concept de « droit global ».

${ }^{4}$ G. TIMSIT, « Système », in Dictionnaire de la culture juridique, op. cit., p. 1462.

${ }^{5}$ Ibid.

${ }^{6}$ Réf. à N. LUHMANN, « Le droit comme système social », Dr. et société 1994, p. 53 s.

${ }^{7}$ Réf. à G. Teubner, Le droit, un système autopoïétique, Puf, coll. Les voies du droit, 1993.

${ }^{8}$ Réf. à R. DAVID, C. JAUFFRET-SPINOSI, Les grands systèmes de droit contemporains, $11^{\mathrm{e}}$ éd., Dalloz, coll. Précis, 2002.

${ }^{9}$ S. Romano, L'ordre juridique (1946), trad. L. François, P. Gothot, Dalloz, coll. Philosophie du droit, 1975, passim.

${ }^{10}$ J.-J. SUEUR, «Analyser le pluralisme pour comprendre la mondialisation ?», in J.-Y. CHÉROT, B. FRYDMAN, dir., La science du droit dans la globalisation, Bruylant, coll. Penser le droit, 2012, p. 96.

${ }^{11}$ Ibid., p. 94.

12 J.-F. AkAndjI-Kombé, « Propos introductifs », in L. FonTAIne, dir., Droit et pluralisme, NemesisBruylant, coll. Droit et justice, 2007, p. 11. 
nous en servons pour désigner quelque-chose d'autre, que nous ne pouvons nommer, mais dont nous sentons bien qu'il n'est qu'une vague approximation ${ }^{1}$. Sans doute pareille analyse peut-elle être étendue à la plupart des concepts polysémiques contemporains précédemment cités.

Enfin, il faudrait sans doute citer à comparaître devant le tribunal du sens le concept de droit en personne. À mesure que la pensée postmoderne subroge la pensée moderne et tend à effacer les lignes de force de celle-ci, la définition du droit se fait plus évanescente encore qu'elle ne l'était déjà. Si le droit est « la catégorie de dispositions légales et de décisions juridictionnelles que les juristes [...] désignent par "droit" "2, la difficulté est que les propositions relatives à cette catégorie sont par trop multiples et qu'il n'est même pas certain, au sens de beaucoup, que le droit soit ainsi un ensemble de «dispositions légales et de décisions juridictionnelles ». Le temps de la domination du normativisme kelsénien est en passe de s'achever, mais aucune théorie générale du droit ne semble en mesure de s'asseoir dans le fauteuil laissé vacant ${ }^{3}$. Et le concept d' «État » n'est peut-être pas mieux loti que celui de «droit » dès lors qu'on le décrit comme « vague et obscur ${ }^{4}$.

À l'aune de ces quelques témoignages et attestations de polysémie, apparaît combien, aujourd'hui plus que jamais, prévenir le développement de l'équivocité dans le discours doctrinal semble être une condition essentielle de la préservation de son aspect scientifique. Plus les concepts auront des allures de «décepts », i.e. de termes «tellement surchargés de significations qu'ils en perdent toute signification $»^{5}$, et moins il sera légitime de qualifier l'activité des juristes de « science » du droit.

\section{B. La lutte contre les concepts polysémiques, enjeu de la science juridique contemporaine}

Beaucoup des concepts postmodernes à la signification incertaine procèdent d'une logique inverse par rapport à celle dont relèvent les concepts propres à la modernité : alors que ces derniers ont été façonnés par quelques auteurs avant de prospérer dans le public des juristes aux côtés d'un sens unique par eux arrêté, les

${ }^{1}$ J.-J. SUEUR, «Analyser le pluralisme pour comprendre la mondialisation ? », in J.-Y. CHÉROT, B. FRYDMAN, dir., La science du droit dans la globalisation, Bruylant, coll. Penser le droit, 2012, p. 94.

${ }^{2}$ Ch. Atias, Science des légistes, savoir des juristes, $3^{\mathrm{e}}$ éd., Presses universitaires d'Aix-Marseille, 1993, p. 10 .

${ }^{3}$ C'est pourquoi l'auteur de ces lignes propose d'adopter une posture syncrétique par rapport aux notions de «droit», de «norme juridique» et «institution juridique». Cf. B. BARRAUD, « L'échelle de juridicité : un outil pour mesurer le droit et fonder une théorie syncrétique », Arch. phil. droit 2013, p. $365 \mathrm{~s}$.

${ }^{4}$ P. KAHN, L'État, Quintette, coll. Philosopher, 1989, p. 5.

${ }^{5}$ P. Musso, Critique des réseaux, Puf, coll. La politique éclatée, 2003. 
concepts tels que «gouvernance » ou «droit souple» se développent — du reste assez inexplicablement - dans la psyché juridique collective et, une fois que tous les juristes les usent en les attachant à des sens multiples et parfois antagonistes, on songe à l'utilité de mener une recherche synthétique dans le but de dégager un sens unitaire. Mais il est alors trop tard et seuls le temps et la patience peuvent permettre aux significations surabondantes de s'estomper puis de s'effacer pour laisser place à une signification principale puis à une signification unique (ou, du moins, proche de l'unité car n'exigeant plus qu'un effort d'interprétation et de compréhension minimal). Ordinairement, la rationalisation scientifique d'un concept précède sa popularisation ou « commercialisation », ce qui est conforme à une certaine logique linguistique puisque, en temps normal, le chercheur est tenté de recourir à un concept en premier lieu parce que celui-ci est rationnel, pertinent, légitime, donc «efficace »scientifiquement. Mais, actuellement, le fonctionnement de la doctrine juridique semble en proie à quelques défaillances qui, peut-être, sont annonciatrices d'une crise plus profonde à venir. Lorsque, par exemple, on entend élaborer et promouvoir le concept de «force normative », on recourt à un ouvrage collectif dans lequel la plupart des quelques soixante contributions comprennent ce concept d'une manière autonome et particulière ${ }^{1}$. La transition de la modernité à la postmodernité de la culture juridique pourrait difficilement faire l'économie d'un temps plus ou moins long de crise, à mesure de la poussée des postmodernes et de la résistance des modernes. Mais les causes du trop-plein de termes aux sens incertains semblent devoir être recherchées surtout dans les difficultés méthodologiques qui marquent nombre de travaux importants, notamment en termes de construction et d'expression - ainsi que de réception — des définitions. Au-delà, la polysémie s'explique déjà par la volonté compréhensible de nombre d'auteurs de s'éloigner des sentiers battus et d'ouvrir de nouvelles voies au sein de l'habillage terminologique du droit, $a$ fortiori en des temps marqués par de grands mouvements.

En tout cas l'attitude des membres de la doctrine juridique est-elle à interroger — si ce n'est à dénoncer - dès lors qu'ils en viennent, face aux idées de «droit mou » et de «droit souple », à exiger que leur soient proposées des «expressions plus englobantes $»^{2}$, ainsi que des concepts «poreux et expansifs $»^{3}$, et que leur soit concédée une « marge d'incertitude dans le vocabulaire » ${ }^{4}$, alors qu'il faudrait exiger à l'inverse des expressions plus précises et sémantiquement mieux fixées ; ou encore dès lors que, devant un concept de «pluralisme juridique » qui se conjugue au pluriel — « les "pluralismes juridiques" sont aussi nombreux que les personnes qui s'y intéressent $»^{5}-$, ils invitent à penser cette notion pourtant déjà intrinsèquement

${ }^{1}$ C. THIBIERGE, dir., La force normative - Naissance d'un concept, LGDJ-Bruylant, 2009.

${ }^{2}$ C. ThiBIERGE, « Le droit souple - Réflexion sur les textures du droit », RTD civ. 2003, p. 599.

${ }^{3}$ M. XIFARAS, « Après les Théories Générales de l’État : le droit global ? », Jus Politicum 2012, n 8, p. 4.

${ }^{4}$ Ch.-A. MoRAND, « Le droit saisi par la mondialisation - Définitions, enjeux et transformations », in ChA. MoRAND, dir., Le droit saisi par la mondialisation, Bruylant, 2001, p. 81.

${ }^{5}$ Ch. EBERHARD, «Penser la pluralisme juridique de manière pluraliste - Défi pour une théorie interculturelle du droit », Cahiers d'anthropologie du droit 2003, $\mathrm{n}^{\circ} 2$. 
plurielle «de manière pluraliste ${ }^{1}$, plutôt que de chercher à en déterminer une (utopique) acception unifiée. Les législateurs peuvent soutenir que «l'indéterminé et l'incertain [sont] des moyens de s'adapter aux courbures de l'espace et du temps $»^{2}$; les scientifiques du droit, eux, ne devraient guère le pouvoir puisque l'indéterminé et l'incertain sont ascientifiques en ce qu'ils ne satisfont pas l'exigence de précision qui est l'une des premières exigences de toute science. Il semble donc impératif de s'opposer fermement à la thèse selon laquelle «une nouvelle approche, particulièrement adaptée au droit, est celle de la "logique floue", qui utilise des standards et des concepts flexibles $»^{3}$. Tout au contraire, la science du droit ne se nourrit que de rigueur et d'exactitude.

Si les bouleversements que connaît le droit dans son versant positif impliquent nécessairement d'autres bouleversements frappant cette fois son pan doctrinal, car toute science positive est contrainte par les faits qui constituent son objet, par leur teneur et leur évolution, il n'en faut pas moins tout mettre en œuvre afin de limiter l' «épidémie de polysémite » que ces mouvements favorisent. La créativité lexicale (formulation de néologismes) et la liberté lexicale (modification du sens de termes déjà «en vigueur ») doivent se limiter aux mots et expressions dont le vocabulaire scientifique a absolument besoin sous peine de souffrir d'insuffisance, c'est-à-dire à la désignation et à l'explication de phénomènes nouveaux qu'aucun concept moderne ne peut adéquatement désigner ou expliquer. Pour le reste, la fonction du scientifique du droit l'astreint à s'efforcer de ne recourir qu'au champ lexical existant et précisément arrêté. Bien sûr, il n'y aurait jamais eu, dans l'histoire de l'humanité, de langage autre que très primaire si n'avait jamais été mise en œuvre quelque créativité lexicale. Mais le néologisme, à l'aube du XXI ${ }^{\mathrm{e}}$ s., ne présente plus la même utilité qu'à l'aube de l'humanité, quand cet outil de communication hyperperfectionné qu'est le langage verbal restait entièrement à forger. Lorsque la langue scientifique est donnée et composée de termes et expressions aux significations uniques et strictes largement acceptées par la communauté scientifique, seuls ceux-ci ont, dans la mesure du possible, à être usités. Les juristes, à l'heure où le droit mue en profondeur et constamment, ont donc à recourir à une créativité et une liberté lexicales modérées et réfléchies, ne servant pas à faire œuvre lyrique, littéraire ou poétique mais seulement à combler les quelques vides et corriger les quelques imperfections de l'ossature conceptuelle moderne. Par exemple, la métaphore, ontologiquement mystérieuse, n'est certainement pas la plus pertinente des unités linguistiques à la disposition des auteurs et évoquer la «constitutionnalisation du droit de l'OMC » ou la «Constitution de l'internet » ${ }^{4}$ revient à recourir à un

${ }^{1}$ Ibid.

${ }^{2}$ M. Delmas-Marty, «La grande complexité juridique du monde», in Mélanges Gérard Timsit, Bruylant, 2004, p. 93.

${ }^{3}$ J.-L. BERGEL, « Méthodologie juridique », in Dictionnaire de la culture juridique, op. cit., p. 1024.

${ }^{4}$ G. Teubner, «Societal Constitutionalism: Alternatives to State Centred Constitutional Theory? », in C. Joerges, I.-J. SAnd, G. Teubner, dir., Constitutionalism and Transnational Governance, Hart Publishing, 2004, p. 3. 
vocabulaire ambigu plongeant le lecteur ou l'auditeur juriste dans l'incertitude, si ce n'est dans la perplexité. En somme, l'idée de «nouvelle modernité » paraît préférable à celle de « postmodernité » qui implique la rupture plus que l'adaptation.

Si la controverse scientifique est indispensable à la science - elle est ce qui lui donne vie et ce qui lui donne le mouvement - , elle n'est possible qu'à condition que les différentes parties prenant part au débat attachent de mêmes signifiés aux signifiants qu'ils emploient. Une controverse virant à la cacophonie ne peut déboucher sur une avancée scientifique; elle ne peut qu'engendrer un «chaos conceptuel»dont il ne semble pas possible d'estimer qu'il «fait sens » ${ }^{1}$. Ainsi l'affirmation de l'impérieuse exigence de monosémie vient-elle poursuivre l'ouvrage kelsénien consistant à «élever la théorie du droit [...] au niveau et rang d'une véritable science» en poursuivant «l'idéal de toute science: l'objectivité et l'exactitude $»^{2}$; car il n'est nulles objectivité et exactitude envisageables sans stricte précision et acceptation des signifiés accolés aux signifiants constituant le langage scientifique. Il est interdit, dans le «laboratoire de recherche» au sein duquel Hauriou disait travailler ${ }^{3}$, de laisser entrer l'incertitude, le vague et le flou qui forment pourtant le cadre dans lequel certains juristes contemporains souhaitent explicitement s'inscrire.

Les législateurs peuvent, à bon droit, user volontairement de concepts polysémique afin de produire un droit positif plus adaptable aux soubresauts de la réalité sociale qu'ils ont vocation à régir ; et Georges Vedel soulignait combien l'équivoque est parfois une arme législative opportune lorsqu'elle permet à chacun des intérêts antagonistes en cause de voir dans le terme ou dans l'expression problématique ce qu'il a envie d'y voir ${ }^{4}$. En revanche, le langage scientifique devrait être un langage qui s'impose au chercheur et qui lui permet de «parler avec méthode, compétence et sagesse », ainsi que le décrivait Montaigne ${ }^{5}$. Selon la première phrase du Discours de la méthode de Descartes, «le bon sens est la chose du monde la mieux partagée $»^{6}$; mais il est fort contestable que, concernant le «bon sens des mots », cette assertion soit vérifiable. Aussi tous les scientifiques, utilisateurs d'un langage scientifique, doivent-ils chercher à participer de la clarification de leur arsenal conceptuel, le «bon sens » étant le «sens unique ».

\footnotetext{
${ }^{1}$ Réf. à G. TUSSEAU, «Un chaos conceptuel qui fait sens : la rhétorique du constitutionnalisme global », in J.-Y. ChÉRot, B. Frydman, dir., La science du droit dans la globalisation, Bruylant, coll. Penser le droit, 2012, p. $182 \mathrm{~s}$.

${ }^{2}$ H. KelSEN, Théorie pure du droit, $2^{\mathrm{e}}$ éd., trad. Ch. Eisenmann, Dalloz, 1962, p. VII.

${ }^{3}$ M. Hauriou, Principes de droit public à l'usage des étudiants en licence et en doctorat ès sciences politiques, $2^{\mathrm{e}}$ éd., Sirey, 1916, p. VII.

${ }^{4}$ G. VEDEL, « De l'arbitrage à la mystique », Preuves 1960, n 112, p. 17.

${ }^{5}$ M. DE Montaigne, Les essais, 1588, L. III, chap. 8.

${ }^{6} \mathrm{R}$. DesCARTES, Discours de la méthode - Pour bien conduire sa raison, et chercher la vérité dans les sciences, 1637.
} 
Avec Carré de Malberg, il faut donc convenir qu' "en matière juridique, la terminologie ne peut être satisfaisante qu'à la condition de comporter un terme propre pour chaque concept spécial. Le danger des mots à double sens, c'est d'amener la confusion dans les idées ${ }^{1}$. Est ici définitivement soutenu que la monosémie est une condition non suffisante mais nécessaire de la scientificité des études juridiques car, si celles-ci laissent place à l'ambivalence, elles se retrouvent dans l'impossibilité de façonner un «ensemble de connaissances ordonnées $»^{2}$. Dans la quête de sens clair et la lutte contre la polysémie résident certains des enjeux majeurs de la science et de la doctrine juridiques contemporaines. Ladite science est parfois désignée comme «vulnérable » en raison de son absence ou, du moins, de son manque de méthode ${ }^{3}$. Tout mettre en œuvre pour travailler aux côtés d'un ensemble conceptuel précis serait plus qu'une première pierre posée à l'édifice d'une méthode scientifique. À raison, Duguit ne comprenait pas que « l'on ne paraît profond que si l'on est obscur » et que beaucoup considèrent qu'est « pauvre d'esprit [qui] veut que soient bannis des études juridiques les formules creuses et les développements métaphoriques qui ont submergé les études philosophiques ${ }^{4}$. Et l'illustre professeur bordelais de dénoncer «Hauriou, qui, comme son modèle Bergson, affectionne particulièrement le langage métaphorique, si commode pour cacher l'imprécision des idées et le vague de la pensée $»^{5}$. Le juriste-scientifique, à l'instar de tout scientifique, devrait s'efforcer d'être intelligible autant qu'intelligent. Ainsi convient-il de soutenir Duguit et, à travers cet auteur, la clarté de l'expression scientifique, laquelle ne va pas sans l'usage de concepts et, plus largement, d'un vocabulaire précisément arrêtés.

Jean Rivero enseignait qu'une réalité complexe est forcément mieux exprimée par un langage complexe que par un langage simple ${ }^{6}$. Mais ladite complexité ne doit pas impliquer l'ambiguité ; elle doit être la conséquence d'un effort de précision. Et reste que, du point de vue du récepteur du discours, la monosémie va de pair avec la simplicité et la limpidité quand la polysémie est surtout synonyme de complexité et d'incompréhensibilité. Par suite, le langage scientifique peut néanmoins pêcher par excès de simplicité et, ainsi que le notait Paul Valéry, "ce qui est simple est faux ; ce qui est complexe est inutile ». Comme souvent, tout n'est en définitive, en la matière, que question d'équilibre et la simplicité de la forme doit rendre justice de la

${ }^{1} \mathrm{R}$. CARRÉ DE MALBERG, Contribution à la théorie générale de l'État spécialement d'après les données fournies par le droit constitutionnel français, t. I, Librairie du Recueil Sirey, 1920, p. 86.

${ }^{2} \mathrm{~V}^{\circ}$ «Science », in A. LALANDE, Vocabulaire technique et critique de la philosophie, Puf, coll. Quadrige dicos poche, 2010.

${ }^{3}$ F. ROUviÈre, «La vulnérabilité de la science du droit: histoire d'une science sans méthode », in F. RouvièRE, dir., Le droit à l'épreuve de la vulnérabilité - Études de droit français et de droit comparé, Bruylant, coll. CRJFC, 2011, p. 537 s.

${ }^{4}$ L. Duguit, Traité de droit constitutionnel - Tome premier : La règle de droit, le problème de l'État, $3^{\mathrm{e}}$ éd., De Boccard, 1927, p. XVI.

5 Ibid., p. 30.

${ }^{6}$ J. RIVERO, « Existe-t-il un critère du droit administratif ? », RDP 1953, p. 292. 
complexité du fond et non la galvauder. Or la meilleure manière de rendre publique simplement une pensée densément construite ou de décrire simplement une réalité complexe consiste à recourir à un langage précis et incontestable - en même temps qu'à un style épuré et fluide - En droit, la simplicité est donc une élégance qui confine à l'exigence.

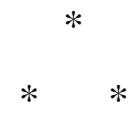

Goethe invitait ses lecteurs à s' «arrêt[er] aux mots » car ils permettraient, selon lui, d' «arriv[er] par la route la plus sûre au temple de la certitude ${ }^{1}$; tandis que, au sens de Hegel, «le langage est le moyen le plus digne de représenter l'esprit $»^{2}$. Mais tout cela n'est possible qu'à condition d'être le fruit d'une délicate et savante entreprise collective et les mots peuvent être tout autant les ennemis de la certitude et de l'esprit, trahissant parfois ce dernier et négligeant d'autres fois cette première. Les concepts sont des moyens de communication très imparfaits; bien souvent, «ils dissimulent au lieu de manifester $»^{3}$. Et il faut beaucoup de temps et de patience pour qu'un concept en bonne et due forme soit affirmé, alors que, rapidement, il peut se trouver dégradé, mis en péril par quelques écrits le travestissant. Aussi n'est-il pas anodin ou accessoire mais bien essentiel de mettre en garde la gent doctrinale contre les dangers inhérents à d'excessives créativité et liberté lexicales. S'il peut être judicieux de chercher à créer de nouveaux concepts lorsque cela apparaît indispensable, il peut être désastreux de le faire quand cela est surabondant par rapport à ce que la nécessité de suivre la mue de l'objet étudié autorise.

«L'histoire, enseignait Bobbio, de la réflexion critique sur la science du droit est celle des modèles qui, à chaque occasion, ont été inventés par les juristes pour accroître la dignité et l'autorité de leur travail, ou pour le rendre plus rigoureux et l'élever ainsi au statut de science $»{ }^{4}$. La présente étude, en entendant mettre en garde les scientifiques du droit contre leur tendance - à l'heure de réviser leur ensemble épistémique et leurs « représentations savantes du droit ${ }^{5}$ à l'aune des mutations des formes du droit positif - à par trop recourir à des concepts polysémiques, aspire à contribuer à cette histoire de la réflexion critique sur la science du droit et, surtout, aspire à participer de l'accroissement de la rigueur, de la dignité et de l'autorité des

${ }^{1}$ J. W. von GoEThe, Faust, trad. G. de Nerval, 1828.

${ }^{2}$ G. W. F. HEGEL, Principes de la philosophie du droit, 1820, § 78.

${ }^{3}$ Ch. Atias, Épistémologie juridique, Dalloz, coll. Précis, 2002, p. 85.

${ }^{4}$ N. BobBio, «Être et devoir-être dans la science du droit», in Essais de théorie du droit, trad. Ch. Agostini, M. Guéret, LGDJ-Bruylant, coll. La pensée juridique, 1998, p. 188.

${ }^{5}$ B. LACROIX, « Transformations du champ juridique et crise dans les représentations savantes du droit », in D. Rousseau, dir., Le droit dérobé, Montchrestien, coll. Grands colloques, 2007, p. 28 s. 
commentaires et pensées juridiques. On a pu qualifier abusivement l'intention de construire une science du droit de «naïve ou simple d'esprit $»^{1}$. En ces lignes, tout au contraire, est affirmé que tant la théorie juridique que la doctrine la plus pragmatique peuvent reposer sur des bases scientifiques profondes et stables; et est souligné combien rien n'est plus décisif pour l'acquisition de ces bases que la qualité du langage, laquelle, sans s'y réduire, dépend de la prévalence des concepts monosémiques par rapport aux concepts polysémiques. A été déjà abondamment signalé à quel point «les sciences sociales, par opposition aux sciences dites “dures", souffrent de leur instabilité » ${ }^{2}$ et, en premier lieu, de l'instabilité de leur langage. Définitivement, il semble que la scientificité d'une activité de recherche soit, ceteris paribus, proportionnelle à la précision et à l'univocité de son vocabulaire qui ne doit pas prendre les traits d'un vulgaire jargon.

Dans la magistrale course à la science des disciplines, la doctrine juridique ne sera jamais rattrapée par la philosophie dont la scientificité est faible, à tel point qu'elle évolue mais ne progresse pas, ainsi qu'en témoigne le fait que Socrate, Platon et Aristote sont aujourd'hui encore les philosophes les plus étudiés tandis que les physiciens et mathématiciens de l'Antiquité sont depuis longtemps dépassés. Et la doctrine juridique ne rattrapera jamais les sciences de la nature, elles seules étant en mesure d'identifier des « vérités scientifiques » en bonne et due forme. Il n'en est pas moins utile de chercher à atteindre le plus haut niveau de scientificité que le particularisme, l'autonomie et l'environnement de la science du droit autorisent. Ainsi, par exemple, « la confection d'une thèse en droit est un travail scientifique et c'est l'efficacité scientifique de l'analyse qui compte $»^{3}$. Or l'efficacité scientifique d'un écrit ne saurait aller de pair avec l'utilisation d'un vocable inefficace, c'est-àdire avec la tenue d'un propos abscons, au contenu et à la portée incertains, nécessitant une interprétation subjective. En outre, dès lors que différents sondages révèlent que $80 \%$ des citoyens font confiance aux scientifiques, tandis que moins de $50 \%$ d'entre eux font confiance au personnel politique et aux journalistes ${ }^{4}$, l'intérêt, pour les juristes, de s'adonner à la science juridique plutôt qu'à la politique juridique ou au journalisme juridique devient patent.

La marche conduisant au langage scientifique parfaitement monosémique demeure néanmoins longue et périlleuse. Mais elle mérite d'être poursuivie, car l'enjeu est de taille : éviter ou, du moins, limiter la crise de la doctrine juridique qui, chaque jour davantage, se laisse ressentir à mesure que les postmodernes et leurs bataillons de concepts postmodernes accentuent la pression exercée sur les modernes et leurs cohortes de concepts modernes. Ainsi que l'écrivait Émile Durkheim, «quand on demande aux gens de se défaire des concepts qu'ils ont l'habitude d'appliquer à un ordre de choses, pour repenser celles-ci à nouveaux frais, on ne

${ }^{1}$ G. ZagrebelsKy, Le droit en douceur (1992), Economica, 1999, p. 12.

${ }^{2}$ M. Grawitz, Méthodes des sciences sociales, $11^{\mathrm{e} e}$ ed., Dalloz, coll. Précis, 2000, $4^{\mathrm{e}}$ de couverture.

${ }^{3}$ R. Romi, Méthodologie de la recherche en droit, $2^{\mathrm{e}}$ éd., Litec, coll. Objectif droit, 2010, p. 45.

${ }^{4}$ Y. GingRas, Sociologie des sciences, Puf, coll. Que sais-je ?, 2013. 
peut s'attendre à recruter une nombreuse clientèle ${ }^{1}$. Pourtant, le temps faisant son œuvre, la clientèle s'accroit petit à petit et bientôt peut-être le clivage de la culture juridique sera-t-il rédhibitoire. Aussi la crainte n'est-elle pas feinte mais réelle : la doctrine juridique semble avancer de plus en plus à tâtons dans le crépuscule précédant une longue nuit d'incertitude, de polémique et de perte du sens du droit. L' « incendie doctrinal» n'est pas une hasardeuse prophétie mais bien une éventualité dont il faut dès à présent chercher à éteindre les premières flammes. La polysémie, que ces pages ont tenté de décrire et de prévenir, compte, parmi ces premières flammes, au nombre des plus incandescentes.

Reste que Nietzsche disait : «Tout penseur profond craint plus d'être compris que d'être incompris ${ }^{2}$. La sentence s'applique-t-elle aux penseurs du droit ? Sans doute car, intrinsèquement, le droit n'est peut-être pas digne de l'idéal scientifique avec lequel on aspire à l'envisager. La polysémie serait alors un voile non pas dévastateur mais salvateur.

\footnotetext{
${ }^{1}$ É. DuRKHeIM, Les règles de la méthode sociologique, $2^{\mathrm{e}}$ éd. (1937), Puf, coll. Bibliothèque de philosophie contemporaine, 1973, p. 144.

${ }^{2}$ F. NIETZSCHE, Par-delà bien et mal, 1886.
} 